\title{
The critical transition of Coulomb impurities in gapped graphene
}

\section{Asorey and A. Santagata}

Centro de Astropartículas y Física de Altas Energías,

Departamento de Física Teórica, Universidad de Zaragoza,

C/ Pedro Cerbuna 12, 50009 Zaragoza, Spain

E-mail: asorey@unizar.es, alessandro.santagata@gmail.com

ABSTRACT: The effect of supercritical charge impurities in graphene is very similar to the supercritical atomic collapses in QED for $Z>137$, but with a much lower critical charge. In this sense graphene can be considered as a natural testing ground for the analysis of quantum field theory vacuum instabilities. We analyze the quantum transition from subcritical to supercritical charge regimes in gapped graphene in a common framework that preserves unitarity for any value of charge impurities. In the supercritical regime it is possible to introduce boundary conditions which control the singular behavior at the impurity. We show that for subcritical charges there are also non-trivial boundary conditions which are similar to those that appear in QED for nuclei in the intermediate regime $118<Z<137$. We analyze the behavior of the energy levels associated to the different boundary conditions. In particular, we point out the existence of new bound states in the subcritical regime which include a negative energy bound state in the attractive Coulomb regime. A remarkable property is the continuity of the energy spectral flow under variation of the impurity charge even when jumping across the critical charge transition. We also remark that the energy levels of hydrogenoid bound states at critical values of charge impurities act as focal points of the spectral flow.

KEYwords: Boundary Quantum Field Theory, Integrable Field Theories, Renormalization Group, Topological States of Matter

ARXIV EPRINT: 2005.04175 


\section{Contents}

1 Introduction 1

2 Charged impurities in graphene 3

2.1 2D Dirac Hamiltonian in a Coulomb background 4

$\begin{array}{lll}2.2 & \text { Boundary conditions for different regimes } & 7\end{array}$

3 Bound states and energy levels $\quad 9$

3.1 Regular regime $\alpha^{2} \leq j^{2}-\frac{1}{4} \quad 11$

3.2 Subcritical regime $j^{2}-\frac{1}{4}<\alpha^{2}<j^{2}$ with $\theta \neq 0$ and $\theta \neq \frac{\pi}{2} \quad 11$

3.3 Subcritical regime $j^{2}-\frac{1}{4}<\alpha^{2}<j^{2}$ with $\theta=0$ (hydrogenoid atom) 12

3.4 Subcritical regime $j^{2}-\frac{1}{4}<\alpha^{2}<j^{2}$ with $\theta=\frac{\pi}{2}$ (meta-hydrogenoid atom) 12

3.5 Critical regime $\alpha^{2}=j^{2}$, for $\theta \neq 0$ and $\theta \neq \pi \quad 12$

3.6 Critical regime $\alpha^{2}=j^{2}$ with $\theta=0($ or $\theta=\pi) \quad 12$

$\begin{array}{lll}3.7 & \text { Supercritical regime } \alpha^{2}>j^{2} & 13\end{array}$

4 Spectral flows of bound states 13

$\begin{array}{ll}\text { 4.1 Spectral flow and boundary conditions } & 13\end{array}$

$\begin{array}{ll}4.2 \text { Spectral flow and impurity charges } & 17\end{array}$

$\begin{array}{llr}5 & \text { Conclusions } & 21\end{array}$

\section{Introduction}

The stability of non-relativistic hydrogenoid atoms is one of the essential features that contributed to consolidate the quantum theory. However in relativistic quantum mechanics there is a critical value of the central point-like charge $Z e$ from where on atom stability is lost [1-4]. This is one of the surprising consequences of relativistic invariance in QED. The phenomenon can be understood in a heuristic way as a falling to the center catastrophe. The critical value in QED is reached when the spectrum of bound states of Dirac equation becomes complex which occurs for $Z>137$. In fact what happens in QED is that when one of the bound states reaches the negative continuum spectrum the vacuum becomes unstable, generating electron-positron pairs. The positron escapes to infinite and the electron screens the central charge. The phenomenon has attracted attention from a fundamental viewpoint because it suggests that could be experimentally tested by detecting an excess of positron in the collisions of heavy nuclei $[5,6]$.

The instability of the atom for supercritical charges has also inspired a new mechanism of quark confinement in QCD [7-11]. The running of the strong coupling under the renormalization group flow in QCD leads to large values of the effective charge of the 
quarks, which reaches very fast supercritical values in the infrared. The instability of the vacuum generates a transition from the perturbative Coulomb regime at short distances to a confinement regime in quarks interactions at large distances [12-15].

The discovery of graphene [16] opened a new window for the analysis of this phenomenon [17]. In that case a similar phenomenon occurs in the presence of charged impurities, but with a much lower critical charge. In graphene the instability yields to a screening of the charge impurity, and the phenomenon has been recently experimentally observed [18]. Motivated by this new physical effect we review the main features of this phenomenon and shed some light in some of its more paradoxical aspects. We address the problem from a viewpoint where quantum unitarity is never lost no matter the strength of charge impurities. In fact we show that the formal analytic continuation of the bounded energy levels of the Coulomb problem into complex values does not mean a loss of Hermiticity of the corresponding effective Hamiltonian. It only shows the existence of non-trivial spectral densities in the continuum spectrum which correspond to the existence of resonances in scattering processes [18].

In order to clarify this issue we analyze in graphene the transition from the subcritical regime to the supercritical one by increasing the values of impurity charges. The results show a continuous behavior of the corresponding energy levels, although the spectral flow is very peculiar: energy levels of hydrogenoid spectrum in the critical regime are focal points of the spectra of subcritical and supercritical regimes. The peculiar behavior of the supercritical regime is reflected by the increasing number of energy levels inside the energy gap, but the continuity of the spectral flow is always preserved along the transitions between the different spectral regimes. Vacuum instability of the corresponding quantum field theory is pointed out by the crossing of the $E=-m$ energy level of the Dirac sea continuum by some eingenvalues of the Dirac Hamiltonian which implies the appearance of pair particle-antiparticle creation mechanism that leads to the screening of the charge impurity.

The analysis of the problem is based in a novel method of dealing with selfadjoint extensions of the Dirac Hamiltonian. In that formalism all cases are approached in an unified and global way that allows to follow the spectral flow of the different (weak-strong) regimes in a smooth way. The analysis can be extended to any space dimension, e.g see [19] for the three dimensional case.

In the section 2 we analyze the unitarity problem of the Dirac Hamiltonian in a Coulomb background. The problem is solved by using the theory of self-adjoint extensions which regularize the singularities associated to the Coulomb potential. The selfadjoint Hamiltonians are classified in different regimes according to the value of impurity charges. In section 3 we calculate the bound states energy spectrum of the Coulomb Hamiltonian in the different regimes. A particular attention is paid to the special cases of hydrogen and meta-hydrogen spectra (see [20,21]), The spectral flow of the bound states spectrum is analysed in section 4, where we also study the analytic properties of this flow in the different subcritical and critical regimes. Finally, the analysis of the results and conclusions is carried out in section 5 . 


\section{Charged impurities in graphene}

Graphene is a two dimensional layer of carbon atoms arranged on a honeycomb lattice of hexagons. The magic of the hexagonal honeycomb structure of graphene leads to a spectral structure in the first Brillouin zone with two contact points $K$ and $K^{\prime}$ (Dirac points) between electronic bands. In a neigbourghood of any of these two points ${ }^{1}$ the spectrum of unbounded electrons is well described in terms of a massless Dirac Hamiltonian [22]

$$
H=-i v_{F}\left(\sigma_{1} \partial_{x}+\sigma_{2} \partial_{y}\right),
$$

where $\sigma_{i}, \mathrm{i}=1,2,3$, are the Pauli matrices and $v_{F}$ is the velocity of the electrons at the Fermi surface, which for suspended graphene is about 300 times smaller than the speed of light in vacuum. This behavior also holds for graphene in a substrate of $\mathrm{SiO}_{2}$ with a slight modification of $v_{F}$.

Although natural graphene behaves like a semi-metal with no spectral gap, for electronic applications it is convenient to open a gap between the bands to reach a semiconductor regime. This behavior can be attained by different methods, either by introducing some disorder or by epitaxially grow graphene on a $\mathrm{SiC}$ substrate [23]. In that case the effective Hamiltonian (2.11) becomes a massive Dirac Hamiltonian

$$
H_{0}=-i v_{F}\left(\sigma_{1} \partial_{1}+\sigma_{2} \partial_{2}\right)+m \sigma_{3},
$$

where $m$ the effective mass of the gap.

In the presence of a charged Coulomb impurity the effective electronic Hamiltonian becomes

$$
H_{m}=-i\left(\sigma_{1} \partial_{x}+\sigma_{2} \partial_{y}\right)+m \sigma_{3}-\frac{\alpha}{r}
$$

where

$$
\alpha=\frac{e_{*}^{2}}{v_{F}}, \quad e_{*}^{2}=\frac{2 e^{2}}{\epsilon+1},
$$

is the effective charge of the impurity, $\epsilon$ the effective dielectric constant of the graphene sheet, $r=\sqrt{x^{2}+y^{2}}$ and the electronic effective speed factor $v_{F}$ has been absorbed by rescaling of coordinates $x=x_{1} / v_{F}, y=x_{2} / v_{F}$. The values of $\alpha$ depend on the substrate where the graphene sheet is grown. For instance, $\alpha \approx 2$ for vacuum, $\alpha \simeq 1$ for $\mathrm{SiO}_{2}$ and $\alpha \simeq 0.35$ for $\mathrm{SiC}$.

The presence of a charge impurity with strong Coulomb interactions generate remarkable effects in the spectroscopic and transport properties. The physics of the effective theory is quite similar to that of relativistic atomic physics where the presence of instabilities is rather well know [1-4]. In any case there is a renewed interest on the theoretical and experimental studies on the Coulomb potential supercritical instabilities [24-39]. The main difference with respect to the 3D analogue (hydrogen-like atoms) is that the value of the supercritical charge is much smaller $\alpha=\frac{1}{2} \ll 137$.

Although the single particle approach to the Coulomb problem constitutes the first step in addressing nontrivial features of the full-fledged many-body interacting theory, most of the phenomenology of graphene physics can be explained from this simplified approach.

\footnotetext{
${ }^{1}$ For simplicity we consider only the $K$ Dirac point and $\hbar=1$.
} 


\subsection{D Dirac Hamiltonian in a Coulomb background}

The presence of a singularity at the origin of the Coulomb potential requires the use of some ultraviolet renormalization mechanism. For such a reason it is convenient to introduce an ultraviolet cut-off $r_{<}>0$ around that singular point $r=0$ and later on take the appropriate limit to extend the Hamiltonian to the whole space $\mathbb{R}^{2} \backslash\{0\}[1,40,41]$. The only physical requirement is unitarity of time evolution, which is equivalent to the self-adjointness of the Hamiltonian defined in such limit. If we exclude from the physical space a disk $D\left(r_{<}\right)=\left\{x \in \mathbb{R}^{2} ;\|x\|<r_{<}\right\}$of radius $r_{<}$around the origin, the most general boundary conditions that preserve selfaldjointness of the Hamiltonian (2.3) are given by $[42-44]$

$$
(1+\not \mathbf{p}) \psi\left(r_{<}\right)=U\left(r_{<}\right) \sigma_{3}(1-\not \mathbf{p}) \psi\left(r_{<}\right),
$$

in terms of a unitary operator $U\left(r_{<}\right)$defined on the boundary values of spinors $\psi\left(r_{<}\right) \in$ $L^{2}\left(S_{r_{<}}^{1}, \mathbb{C}^{2}\right)$, where $\mathbf{n}$ denotes the normal vector to the circumference $S_{r_{<}}^{1}=\left\{x \in \mathbb{R}^{2} ;\|x\|=\right.$ $\left.r_{<}\right\}$. This fact derives from the general theory of selfadjoint extensions developed by von Neumann and Krein [44]. Roughly speaking, up to technicalities, the boundary conditions must cancel the boundary term

$$
\int_{S_{r_{<}}^{1}} \psi_{1}^{\dagger} \not \mathbf{q} \psi_{2}
$$

obtained by integrating by parts the matrix element

$$
\left\langle\psi_{1}\left|H_{m}\right| \psi_{2}\right\rangle-\overline{\left\langle\psi_{2}\left|H_{m}\right| \psi_{1}\right\rangle},
$$

and the boundary conditions (2.4) define the maximal linear subspaces of the domain of $H_{m}^{\dagger}$ where the boundary term (2.5) vanishes.

Using polar coordinates $r$ and $\theta$ a general spinor $\psi$ can be expanded as

$$
\psi(r, \phi)=\sum_{l=-\infty}^{\infty}\left(F_{l}(r) \Phi_{l}^{+}(\phi)+G_{l}(r) \Phi_{l}^{-}(\phi)\right)
$$

in terms of orthogonal eigenfunctions

$$
\Phi_{l}^{+}(\phi)=\left(\begin{array}{c}
e^{i l \phi} \\
0
\end{array}\right) \text { and } \Phi_{l}^{-}(\phi)=\left(\begin{array}{c}
0 \\
i e^{i(l+1) \phi}
\end{array}\right),
$$

of the total angular momentum $J_{z}=L_{z}+S_{z}=-i \frac{\partial}{\partial \phi}+\frac{1}{2} \sigma_{3}$, with semi-integer eigenvalues $j=l+1 / 2$. The space of spinors can be then decomposed as orthogonal sum of subspaces with fixed total angular momentum $j \in \mathbb{Z}+\frac{1}{2}$ :

$$
\psi=\sum_{j} \psi_{j}
$$

where $\psi_{j}$ is a spinor of the form

$$
\psi_{j}(r, \phi)=\left(\begin{array}{c}
F^{j}(r) e^{i(j-1 / 2) \phi} \\
i G^{j}(r) e^{i(j+1 / 2) \phi}
\end{array}\right),
$$

which belongs to the subspace of total angular momentum $j$, 
In order to preserve the $\mathrm{SO}(2)$ rotation symmetry in the regularized theory, the unitary operator $U\left(r_{<}\right)$fixing the boundary condition has to be diagonal in the angular momentum decomposition (2.6),

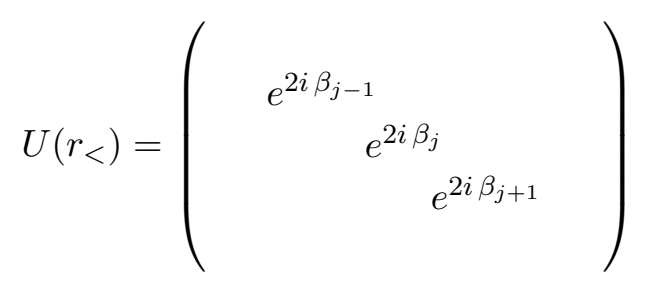

i.e. on each subspace of fixed angular momentum $j$ the unitary operator $U\left(r_{<}\right)$reduces to a single phase $e^{2 i \beta^{j}}$. Thus, the boundary condition (2.4) becomes

$$
(1+\not \mathbf{p}) \psi_{j}\left(r_{<}\right)=e^{2 i \beta^{j}\left(r_{<}\right)} \sigma_{3}(1-\not \mathbf{p}) \psi_{j}\left(r_{<}\right)
$$

More explicitly,

$$
e^{2 i \beta^{j}\left(r_{<}\right)}=\frac{F^{j}\left(r_{<}\right)+i G^{j}\left(r_{<}\right)}{F^{j}\left(r_{<}\right)-i G^{j}\left(r_{<}\right)}
$$

where $F\left(r_{<}\right)$and $G\left(r_{<}\right)$are real functions.

The removal of the UV regularization requires to take the limit $r_{<} \rightarrow 0$ which implies the choice of an appropriate series of boundary conditions $U\left(r_{<}\right)$. The optimal choice of boundary conditions $U\left(r_{<}\right)$that guarantees the convergence of the UV limit is given by the flow driven by asymptotic zero modes. Near the impurites asymptotic zero modes are solutions of the equation

$$
\left[-i\left(\sigma_{1} \partial_{x}+\sigma_{2} \partial_{y}\right)-\frac{\alpha}{r}\right] \psi_{0}=0
$$

They will play a fundamental role in the renomalization of the singularity introduced by the impurities as they do in the three-dimensional case of hydrogenoid atoms $[19,45,46]$. The key observation is that in the vicinity of the inpurity $0<r \ll r_{<}$any solutions of the Coulomb-Dirac equation $H \psi=E \psi$ behaves as an asymptotic zero mode. Thus, all the spinors in the domain of the Hamiltonian must behave near the singularity as zero modes of (2.11).

For any choice of boundary condition $\beta_{0}^{j}$ at a given cut-off $r_{0}$ there is a unique asymptotic zero mode $\left(F_{0}^{j}, G_{0}^{j}\right)$ satisfying the equation

$$
e^{2 i \beta_{0}^{j}}=\frac{F_{0}^{j}\left(r_{0}\right)+i G_{0}^{j}\left(r_{0}\right)}{F_{0}^{j}\left(r_{0}\right)-i G_{0}^{j}\left(r_{0}\right)} .
$$

If the two components of the asymptotic zero mode $\left(F_{0}^{j}, G_{0}^{j}\right)$ are $L^{2}$ normalizable in a neigbourghood of the singularity, i.e. $F_{0}^{j}, G_{0}^{j} \in L^{2}\left(D\left(r_{<}\right), \mathbb{R}\right)$, then the flow of boundary conditions $\beta_{r<}^{j}\left(r_{<} \in\left(0, r_{0}\right)\right)$ given by

$$
e^{2 i \beta^{j}\left(r_{<}\right)}=\frac{F_{0}^{j}\left(r_{<}\right)+i G_{0}^{j}\left(r_{<}\right)}{F_{0}^{j}\left(r_{<}\right)-i G_{0}^{j}\left(r_{<}\right)},
$$


defines in the limit $r_{<} \rightarrow 0$ a selfadjoint extension of the Dirac Hamiltonian (2.3). The domain of the Hamiltonian is expanded by the spinors $\left(F_{0}^{j}, G_{0}^{j}\right)$ which satisfy

$$
\lim _{r \rightarrow 0}\left(F^{j}(r) G_{0}^{j}(r)-G^{j}(r) F_{0}^{j}(r)\right)=0 .
$$

This formula can easily be derived by combining equations $(2.13)$ and $(2.10){ }^{2}$

In other terms, once the cut-off $r_{0}$ is fixed, we can associate to each boundary condition parametrized by $\beta_{0}^{j}$ a unique asymptotic zero mode satisfying (2.12). The other way around, given an asymptotic zero mode, the relation (2.13) defines for each $r_{<} \in\left(0, r_{0}\right)$ a boundary phase $\beta_{r<}^{j}$ in a unique way.

For some values of the impurity charge not all the boundary conditions $\beta_{0}^{j}$ give rise to normalizable asymptotic zero modes. Such boundary conditions do not lead by the procedure described above to a well defined selfadjoint Dirac Hamiltonian. However, as we shall see later on, it is always possible to find an alternative boundary condition $\widehat{\beta}_{0}^{j}$ for the same value of impurity charge whose zero mode is normalizable and leads to a well defined selfadjoint Dirac Hamiltonian. ${ }^{3}$

By this method we have replaced the convergent flow of UV cut-off boundary conditions just by the choice of a simple asymptotic boundary condition (2.14). The boundary condition flow is then defined in this way: the initial cut-off phase $\beta_{0}^{j}$ defines an asymptotic zero mode $\left(F_{0}^{j}, G_{0}^{j}\right)$, and the boundary phases $\beta^{j}\left(r_{<}\right)$run with the cut-off while keeping fixed the zero mode, converging to a well defined boundary condition when the cut-off is removed.

In summary, the boundary condition of the Dirac Hamiltonian in a Coulomb background when the two components of asymptotic zero modes are $L^{2}$ normalizable is defined by the choice of one of these two equivalent boundary data: either a unitary matrix $U\left(r_{0}\right)$ of the form (2.8) acting on the functions of the boundary of the cut-off disk of radius $r_{0}$ or a normalizable asymptotic zero mode $\left(F_{0}^{j}, G_{0}^{j}\right)$. The connection between the two choices is given by equation (2.4). Moreover, any boundary condition that leads to selfadjoint extension of the Hamiltonian (2.13) is obtained by this method.

Thus, the most general boundary conditions in such regimes depend on a UV scale $r_{0}$ and a dimensionless angular parameter $\beta_{j}$ [42-44]. In those cases, the space of boundary conditions has the topology of an infinity cylinder $S^{1} \times \mathbb{R} \equiv \mathbb{R}^{2} \backslash\{0\}$ for any value of the impurity charge $\alpha$. The physical meaning of the boundary conditions can be associated to the insertion of a repulsive $\delta$ function potencial with $r_{0}$ strength. This potential is enough to stabilize the falling to the center trend of strong attractive Coulomb potentials. The dimensionful nature of $r_{0}$ becomes evident from this physical interpretation. In the case where there is no freedom in the choice of boundary conditions is because the Coulomb potential is not strong enough to attract to the electrons to the center which make the system

\footnotetext{
${ }^{2}$ The boundary conditions are independent of the mass $m$ of the electrons. In fact they also hold for massless particles. Notice that $m$ does not appear in the asymptotic equation (2.11) which defines the asymptotic zero-modes.

${ }^{3}$ In any case one can define alternative prescriptions of the boundary conditions flows which starting from a non-normalizable boundary condition converge to the trajectories of normalizable boundary conditions. However, these prescriptions are not canonical and will not be consider here.
} 
insensitive to the presence of a $\delta$ function perturbation. The dependence of the boundary conditions on a dimensionful parameter $r_{0}$ has very relevant physical implications. In the massless limit it implies the breaking of conformal invariance due to the choice of boundary conditions. This phenomenon which is also present in QCD provides in that case the physical argument for the opening a mass gap and an anomalous breaking of conformal symmetry [12-15].

\subsection{Boundary conditions for different regimes}

The subspace of asymptotic zero modes $\left(F_{0}^{j}, G_{0}^{j}\right)$ satisfying the boundary condition $(2.12)$ for a given angular momentum $j \in \mathbb{Z}+\frac{1}{2}$ depends on the value of the charge $\alpha$ of the impurity, in a similar way as in the three-dimensional analogue case $[19,45,46]$

To find the asymptotic zero modes of the Hamiltonian (2.3) we have to look only at leading terms asymptotic expansion around the impurity. Using the expansion (2.7) is easy to show that they satisfy the following coupled equations

$$
\begin{aligned}
& \frac{d F_{0}^{j}}{d r}-\frac{j-1 / 2}{r} F_{0}^{j}+\frac{\alpha}{r} G_{0}^{j}=0, \\
& \frac{d G_{0}^{j}}{d r}+\frac{j+1 / 2}{r} G_{0}^{j}-\frac{\alpha}{r} F_{0}^{j}=0 .
\end{aligned}
$$

Searching for solutions of the form $F_{0}^{j}(r)=r^{s}$ and $G_{0}^{j}(r)=C r^{s}$ we find two independent solutions

$$
s_{ \pm}=-1 / 2 \pm \nu, \quad C_{ \pm}=(j \mp \nu) / \alpha \quad \text { if } \quad \alpha^{2} \neq j^{2},
$$

where $\nu=\sqrt{j^{2}-\alpha^{2}}$. For $\alpha^{2}=j^{2}$ the two solutions degenerate, but in this case the logarithmic corrections give rise also to two independent solutions of the form

$$
\begin{array}{llrl}
F_{0}^{j}(r) & =r^{-1 / 2} & G_{0}^{j}(r) & =\frac{j}{|j|} r^{-1 / 2} \\
F_{0}^{j}(r) & =r^{-1 / 2} \log (\Lambda r) & G_{0}^{j}(r) & =\frac{j}{|j|} r^{-1 / 2}\left[\log (\Lambda r)-\frac{1}{j}\right],
\end{array}
$$

where $\Lambda=1 / r_{0}$.

Notice that the value $j^{2}=\alpha^{2}$ is critical: when $\alpha^{2}<j^{2}$ the parameter $\nu$ is real, while for $\alpha^{2}>j^{2}$ it is purely imaginary. Thus, depending on the strength of the charge impurity there are three different regimes where to impose the boundary conditions.

a) Regular regime: $\alpha^{2} \leq j^{2}-\frac{1}{4}$. This regime is never reached in the lowest angular momentum states $j= \pm 1 / 2$.

In this case $\nu$ is a real parameter and one of the two asymptotic zero modes solutions is not normalizable in a neigbourghood of the origen $D\left(r_{0}\right)$. Indeed, the asymptotic zero mode

$$
\psi_{j}^{-}(r, \phi)=\left(\begin{array}{c}
r^{s_{-}} e^{i(j-1 / 2) \phi} \\
i C_{-} r^{s_{-}} e^{i(j+1 / 2) \phi}
\end{array}\right) \notin L^{2}\left(D\left(r_{0}\right), \mathbb{C}^{2}\right),
$$


is not square integrable in $D\left(r_{0}\right)$. Thus, we are left with only one asymptotic behaviour given by the normalizable zero mode

$$
\psi_{j}^{+}(r, \phi)=\left(\begin{array}{c}
r^{s_{+}} e^{i(j-1 / 2) \phi} \\
i C_{+} r^{s_{+}} e^{i(j+1 / 2) \phi}
\end{array}\right) \in L^{2}\left(D\left(r_{0}\right), \mathbb{C}^{2}\right),
$$

which strongly constrains the boundary condition (2.10), In particular, the parameter $\beta_{0}^{j}$ is not free, it is completely fixed by the boundary condition $(2.12)$

$$
\beta_{0}^{j}=\frac{1}{2} \arcsin \left|\frac{\alpha}{j}\right|
$$

independently of $r_{0}$. This means that there is a unique self adjoint extension of the Hamiltonian (2.3). The boundary condition (2.14) becomes:

$$
\lim _{r \rightarrow 0}\left[(-j+\nu) F^{j}(r)+\alpha G^{j}(r)\right]=0 .
$$

b) Subcritical regime: $j^{2}-\frac{1}{4}<\alpha^{2}<j^{2}$. In this regime both solutions are normalizable, thus the most general asymptotic zero mode is a linear combination of the two solutions (2.20) (2.21). The choice of $\beta_{0}^{j} \in[0, \pi)$ fixes that linear combination in a unique way, up to a global constant.

$$
\begin{aligned}
& F_{0}^{j}(r)=r^{-1 / 2}\left(\cos \theta(\Lambda r)^{\nu}-\sin \theta(\Lambda r)^{-\nu}\right) \\
& G_{0}^{j}(r)=r^{-1 / 2}\left(\cos \theta C_{+}(\Lambda r)^{\nu}-\sin \theta C_{-}(\Lambda r)^{-\nu}\right),
\end{aligned}
$$

where the parameter $\theta \in[0, \pi)$ of the linear combination is given according to the boundary condition (2.12) by

$$
\tan \theta=\frac{(j-\nu) \cos \beta_{0}^{j}-\alpha \sin \beta_{0}^{j}}{(j+\nu) \cos \beta_{0}^{j}-\alpha \sin \beta_{0}^{j}}\left(\Lambda r_{0}\right)^{2 \nu} .
$$

Thus, the boundary condition (2.14) becomes:

$$
\lim _{r \rightarrow 0}\left[\left(j\left(\tan \theta-(\Lambda r)^{2 \nu}\right)+\nu\left(\tan \theta+(\Lambda r)^{2 \nu}\right)\right) F^{j}(r)-\alpha\left(\tan \theta-(\Lambda r)^{2 \nu}\right) G^{j}(r)\right]=0
$$

c) Critical regime: $\alpha^{2}=j^{2}$. In this case the most general asymptotic zero mode is

$$
\begin{aligned}
& F_{0}^{j}(r)=r^{-1 / 2}(\cos \theta+\sin \theta \log (\Lambda r)) \\
& G_{0}^{j}(r)=r^{-1 / 2} \frac{j}{|j|}\left(\cos \theta+\sin \theta\left(\log (\Lambda r)-\frac{1}{j}\right)\right),
\end{aligned}
$$

where the parameter $\theta \in[0, \pi)$ can be related to the phase $\beta_{0}^{j} \in[0, \pi)$ of the boundary condition (2.12) imposed at $S_{r_{0}}^{1}$

$$
\tan \theta=\frac{j-|j| \tan \beta_{0}^{j}}{1-\log (\Lambda r)\left(j-|j| \tan \beta_{0}^{j}\right)} .
$$

The corresponding boundary condition (2.14) of the Dirac operator becomes:

$$
\lim _{r \rightarrow 0}\left[|j| G^{j}(r)(1+\log (\Lambda r) \tan \theta)-F^{j}(r)(j+[-1+j \log (\Lambda r)] \tan \theta)\right]=0 .
$$


d) Supercritical regime: $\boldsymbol{\alpha}^{2}>\boldsymbol{j}^{2}$. In this case the value of $\nu$ becomes imaginary and both asymptotic zero modes are normalizable. A general zero mode solution is of the form

$$
\begin{aligned}
& F_{0}^{j}(r)=r^{-1 / 2}\left(e^{-i \theta}(\Lambda r)^{\nu}+e^{i \theta}(\Lambda r)^{-\nu}\right) \\
& G_{0}^{j}(r)=r^{-1 / 2}\left(e^{-i \theta} C_{+}(\Lambda r)^{\nu}+e^{i \theta} C_{-}(\Lambda r)^{-\nu}\right),
\end{aligned}
$$

where the parameter $\theta \in[0, \pi)$ is fixed by the phase $\beta_{0}^{j} \in[0, \pi)$ of the boundary condition (2.12) imposed at $S_{r_{0}}^{1}$

$$
e^{2 \theta i}=\frac{(\nu-j) \cos \beta_{0}^{j}+\alpha \sin \beta_{0}^{j}}{(\nu+j) \cos \beta_{0}^{j}-\alpha \sin \beta_{0}^{j}}\left(\Lambda r_{0}\right)^{2 \nu} .
$$

The asymptotic boundary condition (2.14) in this case reads

$$
\lim _{r \rightarrow 0}\left[\left(\nu\left(e^{2 i \theta}-(\Lambda r)^{2 \nu}\right)+j\left(e^{2 i \theta}+(\Lambda r)^{2 \nu}\right)\right) F^{j}(r)-\alpha\left(e^{2 i \theta}+(\Lambda r)^{2 \nu}\right) G^{j}(r)\right]=0 .
$$

Notice that in any of the above regimes the Hamiltonian (2.3) is a selfadjoint operator.

From now on we will parametrize the boundary conditions by $\theta \in(0, \pi)$ and $\Lambda$ keeping in mind its relations with $\beta_{0}^{j}$ and $r_{0}$.

\section{Bound states and energy levels}

Once we have shown that the Dirac Hamiltonian (2.3) is a selfadjoint operator it is possible to analyze its spectrum by finding the energy levels

$$
H \psi=E \psi
$$

The eigenvalue problem can be reduced, by using the ansatz (2.7) for each subspace of fixed angular momentum $j$, to solve the pair of coupled differential equations

$$
\begin{aligned}
& \frac{d F^{j}}{d r}-\frac{j-1 / 2}{r} F^{j}+\left(E+m+\frac{\alpha}{r}\right) G^{j}=0, \\
& \frac{d G^{j}}{d r}+\frac{j+1 / 2}{r} G^{j}-\left(E-m+\frac{\alpha}{r}\right) F^{j}=0 .
\end{aligned}
$$

Let us now introduce two radial functions $a(r)$ and $b(r)$ defined by

$$
F^{j}(r)=\frac{\sqrt{m+E}}{2 r}(a(r)-b(r)), G^{j}(r)=\frac{\sqrt{m-E}}{2 r}(a(r)+b(r)),
$$

and use the notation $\epsilon=\sqrt{m^{2}-E^{2}}, x=2 \epsilon r$. The coupled equations satisfied by $a(x)$ and $b(x)$ become

$$
\begin{array}{r}
x a^{\prime}(x)+\left(\frac{x}{2}-\frac{1}{2}-\frac{\alpha E}{\epsilon}\right) a(x)+\left(\frac{\alpha m}{\epsilon}+j\right) b(x)=0, \\
x b^{\prime}(x)-\left(\frac{x}{2}+\frac{1}{2}-\frac{\alpha E}{\epsilon}\right) b(x)+\left(-\frac{\alpha m}{\epsilon}+j\right) a(x)=0,
\end{array}
$$


which can be easily decoupled. Indeed it is obvious to realize that

$$
b(x)=\frac{\left.(2 \alpha E+\epsilon-\epsilon x) a(x)-2 \epsilon x a^{\prime}(x)\right)}{2(\alpha m+j \epsilon)}
$$

and then

$$
a^{\prime \prime}(x)+\left(-\frac{1}{4}+\frac{\frac{1}{2}+\alpha \frac{E}{\epsilon}}{x}+\frac{\frac{1}{4}-j^{2}+\alpha^{2}}{x^{2}}\right) a(x)=0 .
$$

The general solution of (3.8) can be expressed in terms of Whittaker functions $W$ and M [47, 48]

$$
a(x)=A W(1 / 2+\alpha E / \epsilon, \nu, x)+B M(1 / 2+\alpha E / \epsilon, \nu, x),
$$

where A and B are constants. In the same way we have that

$$
b(x)=(j-\alpha m / \epsilon) A W(-1 / 2+\alpha E / \epsilon, \nu, x)+\left(\frac{\alpha m-j \epsilon}{\alpha E+\nu \epsilon}\right) B M(-1 / 2+\alpha E / \epsilon, \nu, x) .
$$

Thus, the general solution of (3.2) and (3.3) is given by

$$
\begin{aligned}
F^{j}(r)= & \frac{\sqrt{m+E}}{2 r}[A(W(1 / 2+\alpha E / \epsilon, \nu, 2 \epsilon r)-(j-\alpha m / \epsilon) W(-1 / 2+\alpha E / \epsilon, \nu, 2 \epsilon r)) \\
& \left.+B\left(M(1 / 2+\alpha E / \epsilon, \nu, 2 \epsilon r)-\left(\frac{\alpha m-j \epsilon}{\alpha E+\nu \epsilon}\right) M(-1 / 2+\alpha E / \epsilon, \nu, 2 \epsilon r)\right)\right], \\
G^{j}(r)= & \frac{\sqrt{m-E}}{2 r}[A(W(1 / 2+\alpha E / \epsilon, \nu, 2 \epsilon r)+(j-\alpha m / \epsilon) W(-1 / 2+\alpha E / \epsilon, \nu, 2 \epsilon r)) \\
& \left.+B\left(M(1 / 2+\alpha E / \epsilon, \nu, 2 \epsilon r)+\left(\frac{\alpha m-j \epsilon}{\alpha E+\nu \epsilon}\right) M(-1 / 2+\alpha E / \epsilon, \nu, 2 \epsilon r)\right)\right]
\end{aligned}
$$

The asymptotic behavior of these solutions is strongly dependent on the regime of charge impurities.

If $\alpha^{2} \neq j^{2}$ the asymptotic behavior can be derived from the behavior of the Whittaker functions $W$ and $M$ for $r \ll 1$,

$$
\begin{aligned}
& M\left( \pm \frac{1}{2}+\alpha E / \epsilon, \nu, x\right) \cong x^{1 / 2+\nu} \\
& W\left( \pm \frac{1}{2}+\alpha E / \epsilon, \nu, x\right) \cong x^{1 / 2}\left(\frac{x^{\nu} \Gamma[2 \nu]}{\Gamma\left[\frac{1}{2} \mp \frac{1}{2}-\nu-\alpha E / \epsilon\right]}+\frac{x^{-\nu} \Gamma[2 \nu]}{\Gamma\left[\frac{1}{2} \mp \frac{1}{2}+\nu-\alpha E / \epsilon\right]}\right),
\end{aligned}
$$

whereas for $\alpha^{2}=j^{2}$ :

$$
\begin{aligned}
& M( \pm 1 / 2+\alpha E / \epsilon, 0, x) \cong x^{1 / 2} \\
& W( \pm 1 / 2+\alpha E / \epsilon, 0, x) \cong-\frac{x^{1 / 2}}{\Gamma\left[\frac{1}{2} \mp \frac{1}{2}-j E / \epsilon\right]}\left(2 \gamma+\psi\left(\frac{1}{2} \mp \frac{1}{2}-j E / \epsilon\right)+\log x\right),
\end{aligned}
$$

where $\psi(x)=\Gamma^{\prime}(x) / \Gamma(x)$ is the digamma function and $\gamma$ the Euler's constant.

The spectrum of energy levels is also strongly dependent on the regime of charges. Let us focus on the discrete energy spectrum which correspond to bound states. 


\subsection{Regular regime $\alpha^{2} \leq j^{2}-\frac{1}{4}$}

The boundary conditions (2.22) can only be satisfied if the constant $A$ of the general solution (3.12) vanishes. On the other hand bound state spinors $\psi$ have to be $L^{2}\left(\mathbb{R}^{3}, \mathbb{C}^{2}\right)$ normalizable which implies that it must to decay at infinity. Thus, the asymptotic behaviour at $r \gg 1$ of (3.2) and (3.3) must be of the form $\left(F^{j}(r) \cong e^{-\epsilon r}, G^{j}(r) \cong e^{-\epsilon r}\right)$. The implies that the spinors should look like

$$
F^{j}(r)=r^{-1 / 2+\nu} e^{-\epsilon r} f(r), G^{j}(r)=r^{-1 / 2+\nu} e^{-\epsilon r} g(r),
$$

where $f(r)$ and $g(r)$ are two radial functions that are polynomially bounded at infinity. This requirement is satisfied when the expressions

$$
\begin{aligned}
& P_{1}(r)=r^{-1 / 2-\nu} e^{\epsilon r} M(1 / 2+\alpha E / \epsilon, \nu, 2 \epsilon r), \\
& P_{2}(r)=r^{-1 / 2-\nu} e^{\epsilon r} M(-1 / 2+\alpha E / \epsilon, \nu, 2 \epsilon r)
\end{aligned}
$$

reduce to polynomials, or when only $P_{1}(x)$ is a polynomial and $\alpha m=j \epsilon$. Expanding $P_{1}$ and $P_{2}$ it is possible to show that this happens when $-\alpha E / \epsilon+\nu=-n$, with $n=0,1,2, .$. if $j>0$ and $n=1,2, .$. if $j<0$. More explicitly, the spectrum of bound states is given by

$$
E_{n}^{H}=\frac{m}{\sqrt{1+\frac{\alpha^{2}}{\left(n+\sqrt{j^{2}-\alpha^{2}}\right)^{2}}}}, \quad n=\left\{\begin{array}{l}
0,1,2, . . \text { for } j>0 \\
1,2,3, . . \text { for } j<0
\end{array} .\right.
$$

This is the well known hydrogenoid atom spectrum of bound states.

For $\alpha^{2}>j^{2}-\frac{1}{4}$ the boundary conditions (2.24) and (2.26) for $\theta \neq 0$ and $\theta \neq \frac{\pi}{2}$ (we will analyze these two exceptional cases later separately), and (2.28) (for any value of $\theta$ ) are satisfied only if the parameter $B$ of the general solution (3.12) vanishes $B=0$. In that case only terms involving the Whittaker function $W$ survive, which implies that they automatically decays exponentially at infinity.

In this sense the exponential decay $e^{-\epsilon r}$ of bound states means that they are localized around the impurity charge and thus behave as edge states in topological insulators [42, 43].

Notice that in the massless limit the exponential decay $e^{-\epsilon r}$ becomes a pure phase factor $e^{-i E r}$ and the corresponding solution is not localized and in fact belongs to the continuum energy spectrum.

\subsection{Subcritical regime $j^{2}-\frac{1}{4}<\alpha^{2}<j^{2}$ with $\theta \neq 0$ and $\theta \neq \frac{\pi}{2}$}

In that case the boundary conditions (2.24) are satisfied only if

$$
\frac{(\alpha(-E+m)+(-j+\nu) \epsilon)}{(\alpha(E-m)+(j+\nu) \epsilon)} \frac{\Gamma[2 \nu] \Gamma[1-\nu-\alpha E / \epsilon]}{\Gamma[-2 \nu] \Gamma[1+\nu-\alpha E / \epsilon]}=\left(\frac{\Lambda}{2 \epsilon}\right)^{-2 \nu} \tan \theta .
$$

The solution of (3.18) gives the spectrum $E_{n}^{I I}(\theta)$ of bound states in that case. However, in the subcritical regime we have two special cases: $\theta=0$ and $\theta=\frac{\pi}{2}$ where the asymptotic zero modes that defines the boundary conditions reduce to one of the two possible different asymptotic behaviors near the origin. Such cases require a special analysis. 


\subsection{Subcritical regime $j^{2}-\frac{1}{4}<\alpha^{2}<j^{2}$ with $\theta=0$ (hydrogenoid atom)}

The boundary conditions reduce in this case to those of the regular regime 3.1, and then the spectrum is the same as in (3.17), i.e the bound states spectrum is the same as the hydrogenoid atom $E_{n}^{H}$.

\subsection{Subcritical regime $j^{2}-\frac{1}{4}<\alpha^{2}<j^{2}$ with $\theta=\frac{\pi}{2}$ (meta-hydrogenoid atom)}

If $\theta=\frac{\pi}{2}$ the boundary conditions are defined by the asymptotic zero modes characterized by the exponent $s=-\frac{1}{2}-\nu$ and become

$$
\lim _{r \rightarrow 0}\left(-(j+\nu) F^{j}(r)+\alpha G^{j}(r)\right)=0 .
$$

These boundary conditions can be satisfied by setting $A=0$ in the general solutions and making the replacement $\nu \rightarrow-\nu$. Using the same techniques as in the regular case, we get the analytic spectrum of bound states

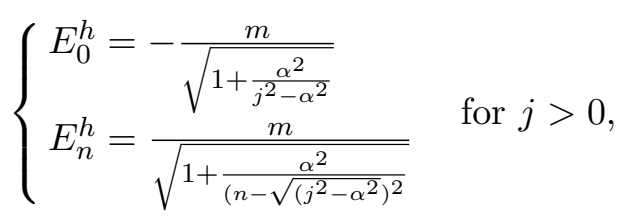

and

$$
E_{n}^{h}=\frac{m}{\sqrt{1+\frac{\alpha^{2}}{\left(n-\sqrt{\left(j^{2}-\alpha^{2}\right)^{2}}\right.}}} \text { for } j<0,
$$

with $n=1,2,3, \ldots$. The above bound states are known meta-hydrogenoid states. ${ }^{4}$ The meta-hydrogenoid bounded spectrum is very similar to the hydrogenoid spectrum. The only difference is a sign in the second radicand.

\subsection{Critical regime $\alpha^{2}=j^{2}$, for $\theta \neq 0$ and $\theta \neq \pi$}

In this case the spectral condition derived from the boundary conditions (2.26) is

$$
\frac{j-|j|\left(m_{\epsilon}-E_{\epsilon}\right)}{\left(j-|j|\left(m_{\epsilon}-E_{\epsilon}\right)\right)\left(2 \gamma-\log \Lambda_{\epsilon} / 2\right)+\left(j-|j| m_{\epsilon}\right) \psi\left(1-|j| E_{\epsilon}\right)+|j| E_{\epsilon} \psi\left(-|j| E_{\epsilon}\right)}=\tan \theta,
$$

where $E_{\epsilon}=E / \epsilon, m_{\epsilon}=m / \epsilon$ and $\Lambda_{\epsilon}=\Lambda / \epsilon$. The solutions of equation (3.22) give an infinite sequence $E_{n}^{I I I}(\theta)$ of discrete energy levels.

\subsection{Critical regime $\alpha^{2}=j^{2}$ with $\theta=0($ or $\theta=\pi)$}

In the critical regime for boundary conditions with $\theta=0$ the hydrogenoid and metahydrogenoid spectra do coincide. They are defined by (3.17) with the only difference that $E_{0}=0$ for $\alpha^{2}=j^{2}$ and $j>0$.

\footnotetext{
${ }^{4}$ The meta-hydrogenoid states first appeared in the literature as hydrino states [20, 21]. However, the misuse of its properties for claiming magic generation of energy requires the introduction of new name. Notice that the hydrogen atom $Z=1, D=3$ is in a subcritical regime where the Hamiltionian is essentially selfadjoint and there is a canonical boundary condition giving rise to the well know spectrum. There is no meta-hydrogen spectrum. Otherwise it will open the interesting window to explain the puzzle of proton radius in an elegant way in terms of more exotic boundary conditions.
} 


\subsection{Supercritical regime $\alpha^{2}>j^{2}$}

The hydrogenoid and meta-hydrogenoid spectra are not well defined for $\alpha^{2}>j^{2}$, the spectral formulae (3.17), (3.20) and (3.21) become complex, but as we have already remarked the spectrum is real and contains an infinite set of discrete energy levels $E_{n}^{I V}(\theta) n \in \mathbb{Z}$ given by the spectral condition

$$
\frac{(\alpha(-E+m)+(-j+\nu) \epsilon)}{(\alpha(E-m)+(j+\nu) \epsilon)} \frac{\Gamma[2 \nu] \Gamma[1-\nu-\alpha E / \epsilon]}{\Gamma[-2 \nu] \Gamma[1+\nu-\alpha E / \epsilon]}=-e^{2 i \theta}\left(\frac{\Lambda}{2 \epsilon}\right)^{-2 \nu} .
$$

\section{Spectral flows of bound states}

The problem which inspired Gribov's approach to confinement is the fact that the energies of the bound states given by $E_{n}^{H}$ become complex for $\alpha^{2}>j^{2}$. To better understand that mechanism let us analyse the flow of the bound state spectrum by continuously increasing the charge $\alpha$ of the impurity or varying the boundary conditions.

\subsection{Spectral flow and boundary conditions}

It is interesting to analyze the flow of the spectrum as we change the boundary condition parameter $\theta$. The continuous flow of the spectrum defined by the change of the parameter $\theta$ characterizing the boundary conditions in the subcritical regime $j^{2}-1 / 4<\alpha^{2}<j^{2}$ is displayed in figure 1 . There we plot for $j=\frac{3}{2}$ the $\theta$ dependence of the lowest energy bound states in this regime.

Notice that in the limits $\theta=0, \theta=\pi / 2, \theta=\pi$ we recover the ydrogenoid and metahydrogenoid spectrum, i.e.

$$
\begin{aligned}
& \lim _{\theta \rightarrow 0} E_{n}^{I I}(\theta)=E_{n}^{H}, \\
& \lim _{\theta \rightarrow \frac{\pi}{2}} E_{n}^{I I}(\theta)=E_{n}^{h} \\
& \lim _{\theta \rightarrow \pi} E_{n}^{I I}(\theta)=E_{n}^{H} .
\end{aligned}
$$

The continuity of the flow should be obvious from the fact that the boundary conditions (2.24) reduce to the boundary conditions of the hydrogenoid and meta-hydrogenoid spectra in these limits. What is more surprising is the fact that the spectral flow is not periodic, i.e. there is an spectral asymmetry. The spectrum is periodic, i.e. it is the same at $\theta$ and $\theta+\pi$, but the flow shifts the energy levels by one unit in each cycle from $\theta=0$ to $\theta=\pi$. In general, for fixed angular momentum and charge we have

$$
E_{n}^{I I}(\theta+k \pi)=E_{n+k}^{I I}(\theta),
$$

for any integer $k \in \mathbb{Z}$. The behaviour of the spectral flow recalls the pumping mechanism exhibited by edge states in topological insulators [49, 50].

An interesting property of the spectral flow is its monotonic behavior, i.e. $E^{I I}(\theta)<$ $E^{I I}\left(\theta^{\prime}\right)$ if $\theta<\theta^{\prime}$. In particular this implies the standard sandwich inequalities between the hydrogenoid and meta-hydrogenoid energy levels

$$
-m<E_{0}^{h}<E_{0}^{H}<E_{1}^{h}<E_{1}^{H}<\cdots<E_{n-1}^{h}<E_{n-1}^{H}<E_{n}^{h}<E_{n}^{H}<\cdots<m .
$$




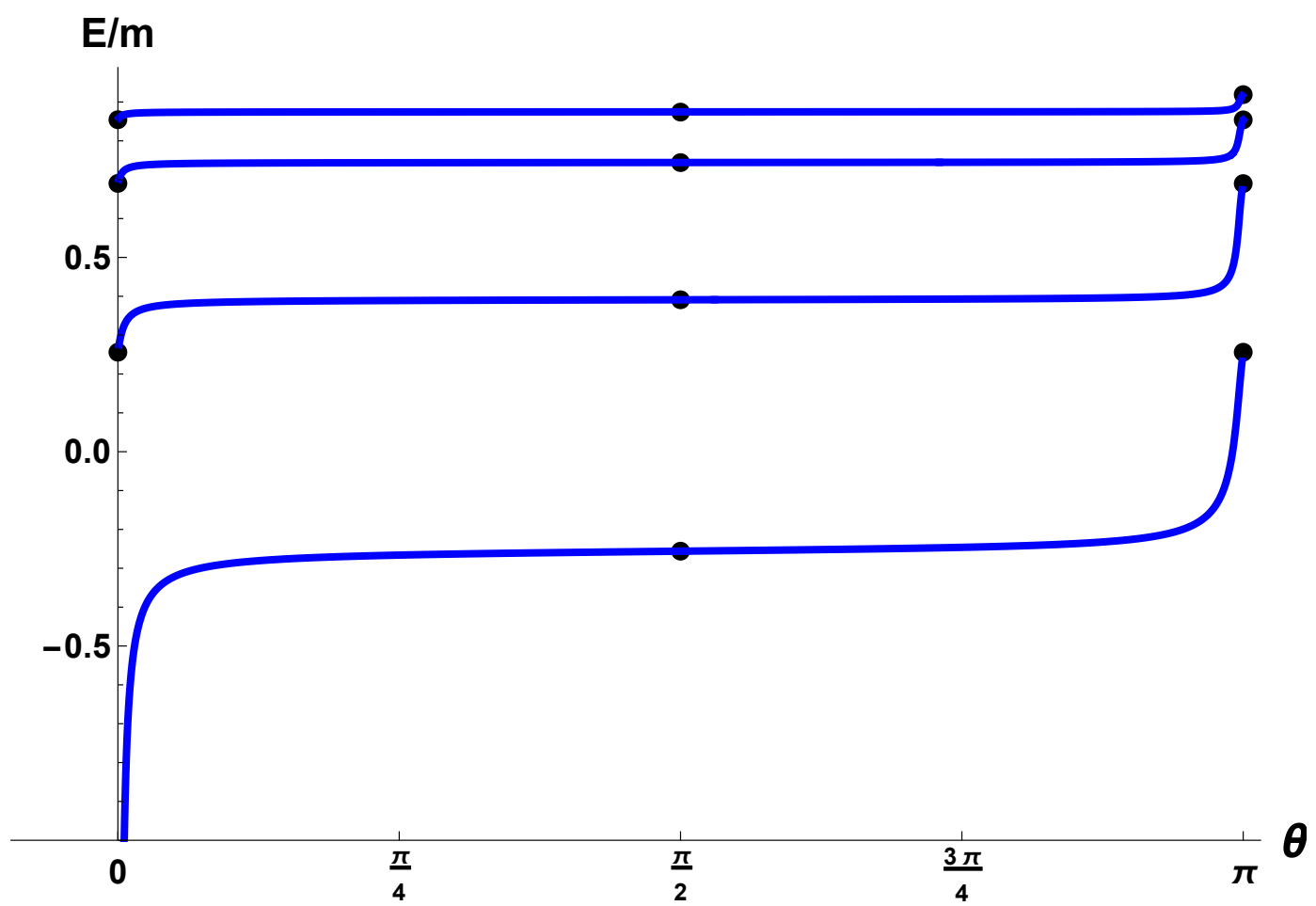

Figure 1. $\theta$ dependence of the energy $E / m$ of the lowest bound states $(n=0,1,2,3)$ with angular momentum $j=3 / 2$ for $\alpha=1.45$ in the subcritical regime $2<\alpha^{2}<\frac{9}{4}(\Lambda=m / 10)$. The dots correspond to the hydrogenoid and meta-hydrogenoid energy levels at $\theta=0, \theta=\frac{\pi}{2}$ and $\theta=\pi$. Notice the $\pi$-periodicity of the spectrum.

Notice that there is a bound state emerging from the continuum $E \leq-m$ at a value of $\theta$ close to $\theta=0$. The behaviour of the flow is the same for positive $j>0$ and negative angular momentum $j<0$, except for the absence of zero levels $(\mathrm{n}=0)$ for the hydrogenoid and meta-hydrogenoid energy levels for $j<0$. Thus the sandwich inequalities in the negative case are

$$
-m<E_{1}^{h}<E_{1}^{H}<\cdots<E_{n-1}^{h}<E_{n-1}^{H}<E_{n}^{h}<E_{n}^{H}<\cdots<m .
$$

Another interesting feature of the subcritical regime is that from (3.17) and (3.21) it follows that for $n>0$ the spectra $E_{n}^{H}$ and $E_{n}^{h}$ with $j>0$ and $j<0$ are degenerate. ${ }^{5}$ The boundary condition (2.24) for $\theta \neq k \pi$ and $\theta \neq \frac{2 k+1}{2}$ breaks this degeneracy and creates a gap between the energies corresponding to $j>0$ and $j<0$.

The situation is described in figure 2 for $Z \alpha=1.45$. For $\theta=0$ we have the energy corresponding to $n=1$ of the hydrogen spectrum $E_{1}^{H}$ which is degenerate for $j= \pm \frac{3}{2}$. As we increase the parameter $\theta$ a gap appears between the states $j= \pm \frac{3}{2}$. The energy of the state $j=-\frac{3}{2}$ becomes lower than the one corresponding to $j=\frac{3}{2}$. The gap disappears again for $\theta=\frac{\pi}{2}$, where we have the again a degenerate energy level corresponding to $n=2$ of the meta-hydrogenoid spectrum $E_{2}^{h}$. If we increase the boundary condition parameter

\footnotetext{
${ }^{5}$ Notice that the same behavior appears in the regular regime (see eq. (3.17)).
} 


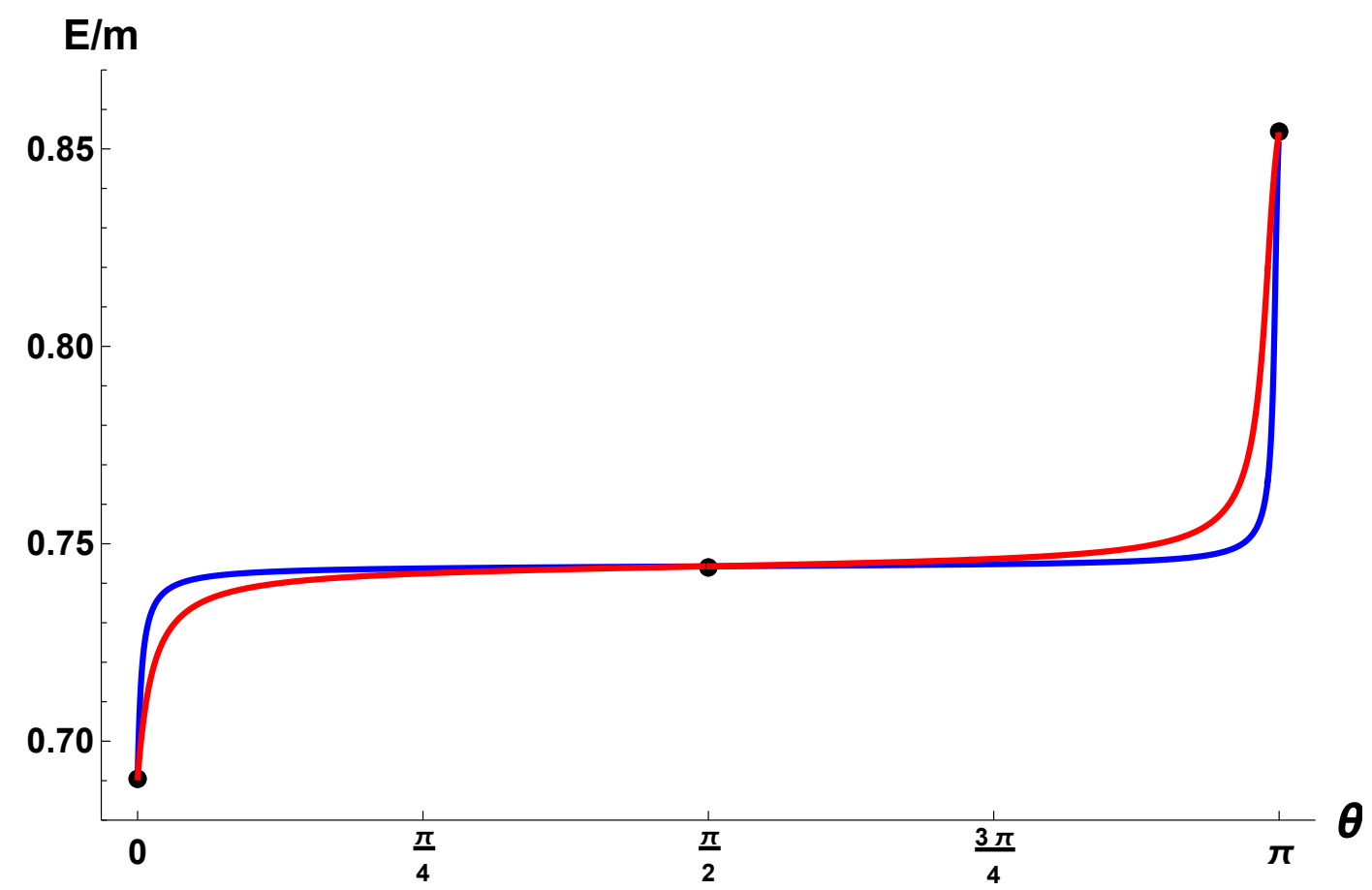

Figure 2. Gap between the energies corresponding to $j=3 / 2$ (blue) and $j=-3 / 2$ (red) for $\alpha=1.45$ and $\Lambda=m / 10$ in the subcritical regime. The flow interpolates from $\theta=0$ to $\theta=\pi$ between the hydrogen levels $n=1$ and $n=2$ and reaches the $n=2$ energy level of meta-hydrogen at $\theta=\pi / 2$.

$\theta$ the gap reappears again. This time with the energy corresponding to $j=\frac{3}{2}$ lower than the one corresponding to $j=-\frac{3}{2}$. Finally, for $\theta=\pi$ the two energy levels become again degenerate at the level $n=2$ of $E_{2}^{H}$.

The origin of this phenomenon resides in the behavior of the theory under time reversal transformations

$$
\boldsymbol{\tau} \psi(x, y, t)=i \sigma_{2} \psi^{*}(x, y,-t) .
$$

The time reversal transformation (4.7) does not leave invariant the Hamiltonian $(2.3)^{6}$

$$
\boldsymbol{\tau} H_{m} \boldsymbol{\tau}=H_{-m}
$$

and the angular momentum operator $J_{3}=x \partial_{y}-y \partial_{x}+\frac{1}{2} \sigma_{3}$

$$
\tau J_{3} \tau=-J_{3}
$$

Some boundary conditions are also not invariant under time reversal tranformation (4.7). In fact the only ones that are time reversal invariant correspond to $\theta=0$ and $\theta=\frac{\pi}{2}$ in the subcritical regime and $\theta=0$ in the critical regime. None of the boundary conditions of the supercritical regime are time reversal imvariant. This behavior is reminiscent of

\footnotetext{
${ }^{6}$ The time reversal symmetry is preserved in the Kane-Mele model [52] of topological insulators which is made of two gapped graphene sheets with opposite masses and without impurities $(\alpha=0) H=H_{m} \oplus H_{-m}$. Actually, many of the interesting features of topological insulators are derived from this invariance.
} 
what happens for scalar fields with boundary conditions which mix normal derivatives and boundary values of the fields [51]. Now, for all boundary conditions where $\boldsymbol{\tau}$ is preserved the spectra of $H_{m}$ and $H_{-m}$ are identical, which implies that for both cases the energy levels with $j>0$ and $-j<0$ are identical. This degeneracy is similar to that of the Kramers effect in topological insulators, except that in this case as $\tau^{2}=\mathbb{I}$ it is not compulsory that all energy levels must be degenerate as in fact it occurs in the case $j=0$ where there is no degeneracy.

In summary the breaking of the $\pm j$ degeneracy for values of $\theta \neq 0, \pi / 2$ is a consequence of the breaking of time reversal symmetry by boundary conditions. This remark have implications for the Kane-Mele model where due to presence of charged impurities the time reversal symmetry can be broken by the same mechanism.

In the critical regime, $\alpha^{2}=j^{2}$, as we have anticipated, the hydrogenoid and metahydrogenoid spectra do coincide with $E_{n}^{I I I}(0)=E_{n}^{I I I}(\pi)$ and are given by (3.17) with the only difference that for $\alpha^{2}=j^{2}$ and $j>0, E_{0}=0$. Once more this fact we can be understood in a simpler way, just by looking at the corresponding boundary conditions. Analyzing how the spectrum $E_{n}^{I I I}(\theta)$ changes with $\theta$, we find that the correspondence in this case is

$$
\begin{aligned}
& \lim _{\theta \rightarrow 0} E_{n}^{I I I}(\theta)=E_{n}^{H}, \\
& \lim _{\theta \rightarrow \pi} E_{n}^{I I I}(\theta)=E_{n}^{h},
\end{aligned}
$$

for $j>0$. The spectrum is also periodic in this case, i.e. it is the same at $\theta$ and $\theta+\pi$, but the flow shifts the energy levels by one unit each time that we increase $\theta$ by $\pi$. In general, for fixed angular momentum and charge we have

$$
E_{n}^{I I I}(\theta+k \pi)=E_{n+k}^{I I I}(\theta),
$$

for any integer $k \in \mathbb{Z}$. But even in that case the spectral flow has a monotonic character, i.e. $E^{I I I}(\theta)<E^{I I I}\left(\theta^{\prime}\right)$ if $\theta<\theta^{\prime}$. The inequalities between the hydrogenoid and metahydrogenoid energy levels (4.6) become the standard inequality of the hydrogenoid levels $E_{n}^{H}<E_{n+1}^{H}$ in this case. The behaviour of the spectral flow recalls again the pumping mechanism of edge states in topological insulators [49, 50].

The degeneracy between the bound energy levels with total angular momentum $j$ and $-j$ (for $n>0$ ) at $\theta=0$ and $\theta=\pi$ is again broken for intermediate values of $\theta \in(0, \pi)$ as shown in figure 3 . The level with negative angular momentum $-|j|$ has always lower energy than that of the corresponding level with positive angular momentum $|j|$. Once more the origin this phenomenon is the breaking of time reversal (or $\mathrm{CP}$ ) invariance by the boundary conditions for $\theta \neq 0, \pi$. The degeneracies at $\theta=0, \pi$ appear by analogous reasons that Kramers effect in topological insulators.

Let us now analyze the supercritical charge regime with $\alpha^{2}>j^{2}$. As anticipated, in this regime, the levels $E_{n}^{H}$ and $E_{n}^{h}$ do not belong to the spectrum of the Hamiltonian. In this case for any value of $\theta$ the energy spectrum $E_{n}^{I V}(\theta)$ contains an infinity number of bound states that accumulate near the mass gap continuum energy level $E=m$. In 


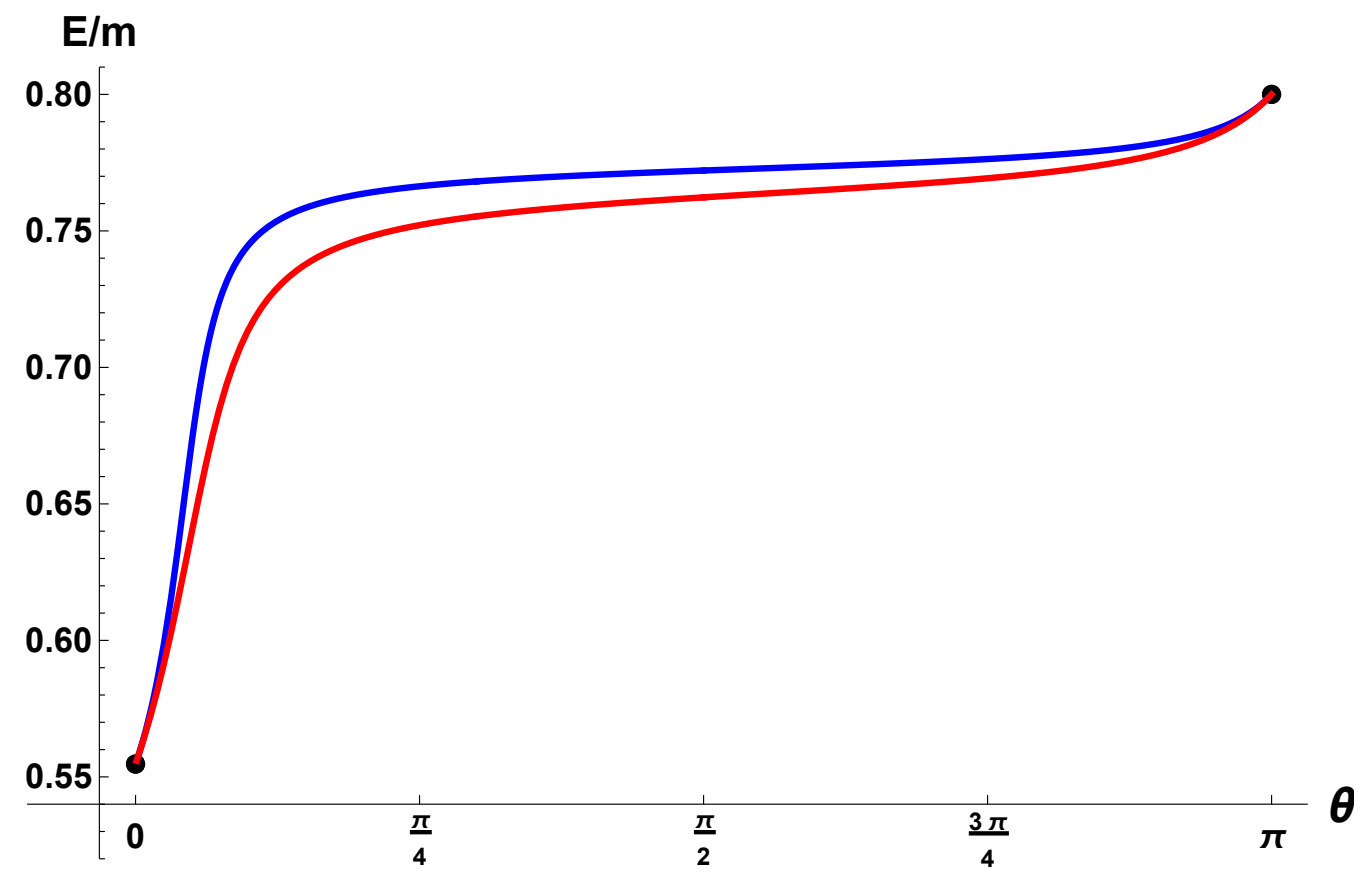

Figure 3. Gap between the energies corresponding to $j=3 / 2$ (blue) and $j=-3 / 2$ (red) for $\alpha=3 / 2$ and $\Lambda=m / 10$ in the critical regime. The flow interpolates from $\theta=0$ to $\theta=p i$ between the hydrogenoid levels $n=1$ and $n=2$.

Figure 4 we plot the flow of some eigenvalues of the spectrum $E_{n}^{I V}(\theta)$ when parameter $\theta$ flows from 0 to $\pi$. Notice that along that flow one eigenvalue pops up from the Dirac sea continuum $E<-m$ at a particular value of the parameter $\theta$.

This is the only footprint of the instabilities pointed out in the supercritical regime, where the analytic expressions of hydrogenoid and meta-hydrogenoid energy levels become formally complex. Notice that the same phenomenon occurs in the subcritical regime $\alpha^{2}<j^{2}$. The appearance of these instabilities is what inspired the Gribov mechanism of quark confinement in QCD [10,11] (see also [12-15]).

\subsection{Spectral flow and impurity charges}

In order to analyze the transition from the subcritical regime to the supercritical regime we fix a suitable value of the parameter $\theta$ for $E_{n}^{I I}(\theta), E_{n}^{I I I}(\theta)$ and $E_{n}^{I V}(\theta)$. By increasing the value of $\alpha$ we can follow the flow of each energy level from the subcritical regime to the critical regime in an adiabatic continuous way. Notice, however, that for each $0<\theta<\frac{\pi}{4}$ there is a bound state in the subcritical regime that merges into the continuum for a special value of $\alpha<j$, and conversely, there is an infinity of bound states emerging from the continuum spectrum for $\alpha \gtrsim|j|$ in the supercritical regime for any $\theta$. In any case we have the following relations

$$
\lim _{\alpha \rightarrow|j|_{-}} E_{n}^{I I}(\theta)=E_{n}^{I I I}(0)=\lim _{\alpha \rightarrow|j|_{+}} E_{n}^{I V}\left(\theta^{\prime}\right)
$$




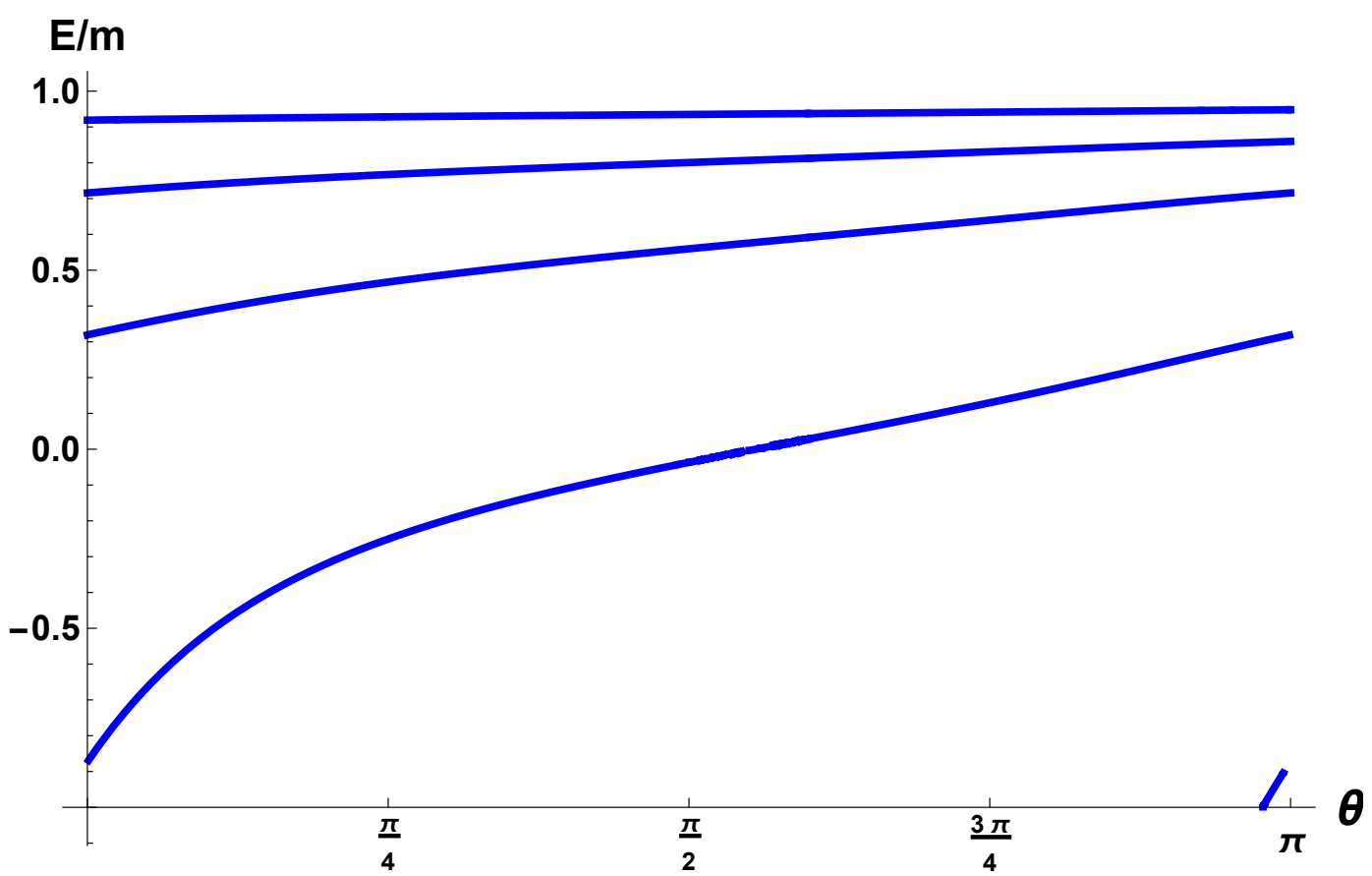

Figure 4. Spectral flow for $\alpha=1.55, \Lambda=m / 10$ and $j=3 / 2$ in the supercritical regime. The lowest bound state energy level emerges from the negative continuum spectrum for a value of $\theta$ close to $\theta=\pi$. Notice the $\pi$-periodicity of the spectrum.

whenever $\theta \neq \frac{\pi}{4}$ and $\theta^{\prime} \neq \frac{\pi}{2}$. This means that, for any fixed values of $\theta\left(\theta \neq \frac{\pi}{4}\right.$ and $\left.\theta^{\prime} \neq \frac{\pi}{2}\right)$, $E_{n}^{I I}(\theta)$ and $E_{n}^{I V}\left(\theta^{\prime}\right)$, converge to $E_{n}^{I I I}(0)$ as $\alpha \rightarrow \alpha_{c}=|j|$, pointing out the continuity of the flow of energy levels in the transition from the subcritical regime to the critical one (See Figure 5). In the exceptional cases we also have continuity in the path crossing the transition border

$$
\lim _{\alpha \rightarrow|j|_{-}} E_{n}^{I I}\left(\frac{\pi}{4}\right)=E_{n}^{I I I}\left(\frac{\pi}{2}\right)=\lim _{\alpha \rightarrow|j|_{+}} E_{n}^{I V}\left(\frac{\pi}{2}\right),
$$

provided we choose the suitable values for the parameter $\theta$ of the boundary condition in the different regimes.

The transition of the spectral flow from the subcritical to the supercritical regime is illustrated in figure 5. The flow shows the dependence of bound states on the impurity charge. They are also highly dependent on the boundary conditions of the different self adjoint extensions. For simplicity we consider the case of angular momentum $j=\frac{3}{2}$ and only a window of the infinite tower of bound states which includes the spectral flow of the lowest bound states of regular and subcritical regimes for different values of $\alpha$. The flow of higher energy levels is in fact very similar. In the region $0<\alpha<\sqrt{2}$, the operator is essentially self adjoint and the spectrum is that of an hydrogenoid atom (black curves in figure 5) that begin at $E=m$ for $\alpha=0$. On the border of the subcritical region $(\alpha=\sqrt{2}$ ) the smallest level (red point in figure 5) is the ground state of the meta-hydrogenoid spectrum, while the other two levels are doubly degenerated, because they include the $n$-level of the hydrogenoid spectrum and the $(n+1)$-level of the meta- 


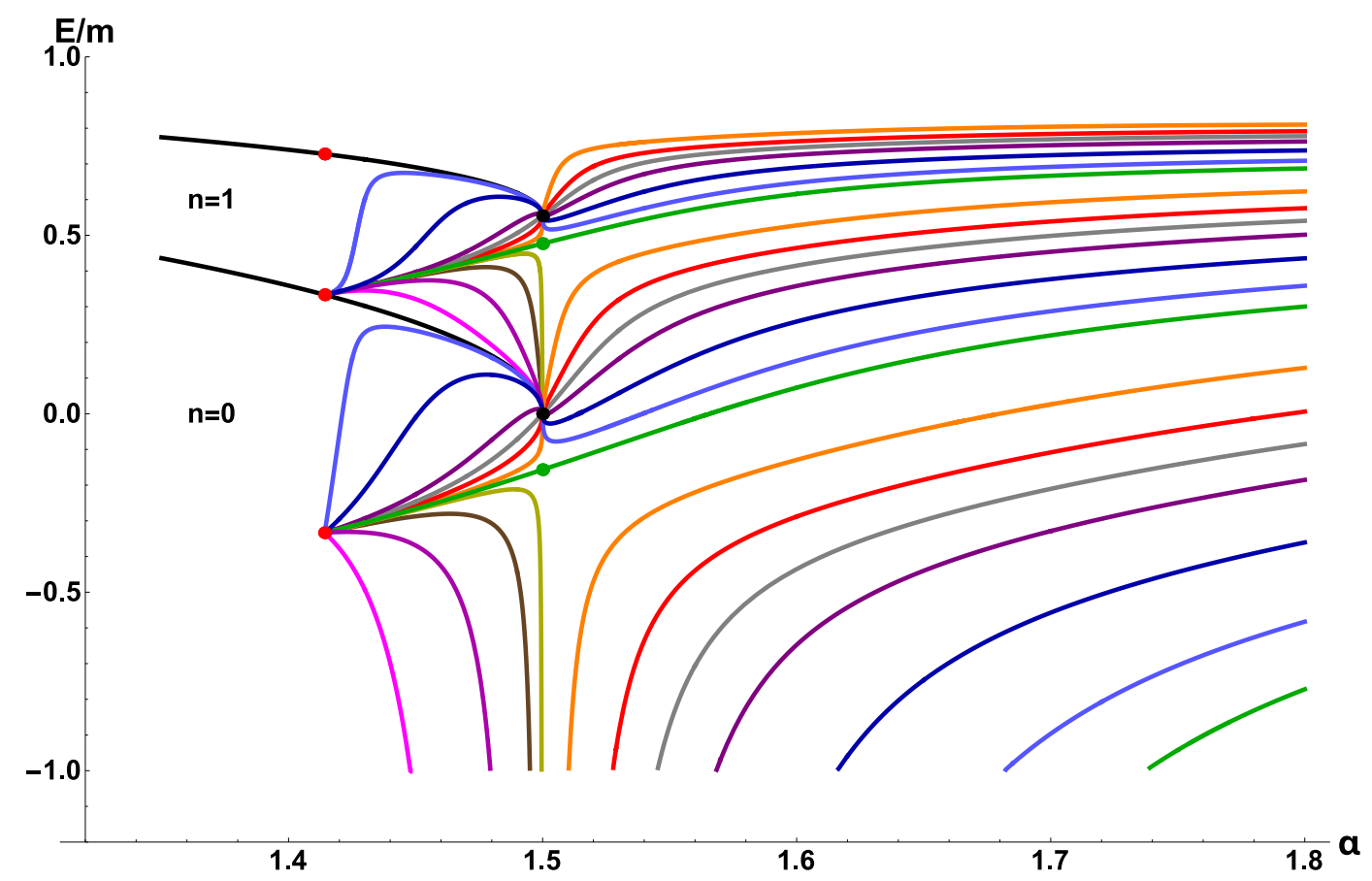

Figure 5. Flow of the lowest energy levels with angular momentum $j=3 / 2(\Lambda=m / 10)$ when the impurity charge crosses from subcritical regime to supercritical regime at $\alpha=3 / 2$. The colors correspond to different choices of boundary conditions. In the subcritical regime $\theta=0$ (hydrogen), $\theta=0.005 \pi, \theta=0.03 \pi, \theta=0.1 \pi, \theta=0.2 \pi, \theta=0.25 \pi$ (isolated), $\theta=0.3 \pi, \theta=0.5 \pi$ (metahydrogen), $\theta=0.75 \pi, \theta=0.9 \pi, \theta=0.99 \pi, \theta=0.999 \pi$; and in the supercritical regime $\theta=0$, $\theta=0.1 \pi, \theta=0.25 \pi, \theta=0.5 \pi$ (isolated), $\theta=0.6 \pi, \theta=0.75 \pi, \theta=0.9 \pi$. Notice that at the critical point $\alpha_{c}=\frac{3}{2}$ the tangent vectors to the spectral curves are vertical except for the green and gray curves which correspond to $\theta=\frac{\pi}{4}$ and $\theta=\frac{3 \pi}{4}$ in the subcritical regime and $\theta=\frac{\pi}{2}$ and $\theta=0$ supercritical regime, respectively.

hydrogenoid spectrum. All the energy levels of the different self adjoint extensions of $H$ start from one of these points.

At the critical coupling $\alpha_{c}=\frac{3}{2}$, we also have some special energy levels, which to some extent, are attractors or repulsors of the other energy levels: the black points correspond to the double degenerate hydrogenoid and meta-hydrogenoid spectra of $\theta=0$, whereas the green points correspond to the bound states of the spectrum of $H$ for $\theta=\pi / 2$. The alternating black and green points are, respectively, stable and unstable fixed points for the flow of energy levels. Each green point attracts only one energy level, that corresponds to the self adjoint extension with $\theta=\pi / 4$ which are on the green curve of figure 5 . These flow curves are isolated and act as repulsive barriers creating bifurcations of the flow. In the subcritical regime for $0 \leq \theta<\pi / 4$ the $n=0$ ground state merges into the continuum flowing to $-\infty$ and each of the other $n>0$ levels flow towards the $n$-th black point, while for $\pi / 4<\theta<\pi$ each of the other $n \geq 0$ levels flow towards the $n+1$ black point (see eq. (4.13)). In the supercritical region, each green point is the starting point of only one level (green curve) which is associated to a particular selfadjoint extension $\theta=\pi / 2$. 


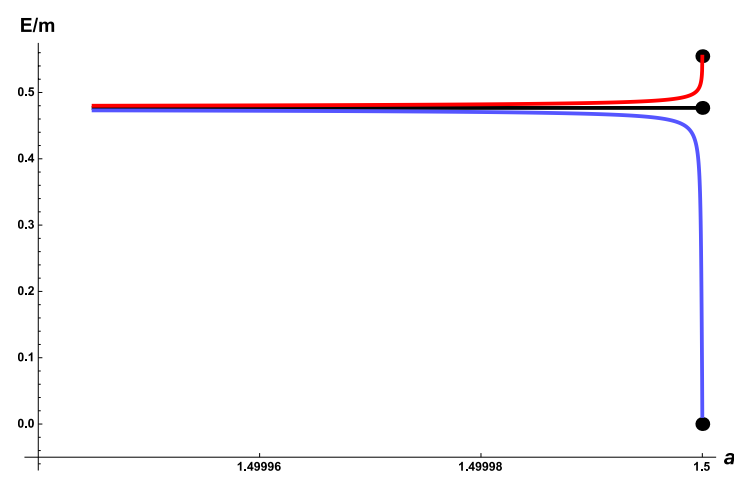

(a)

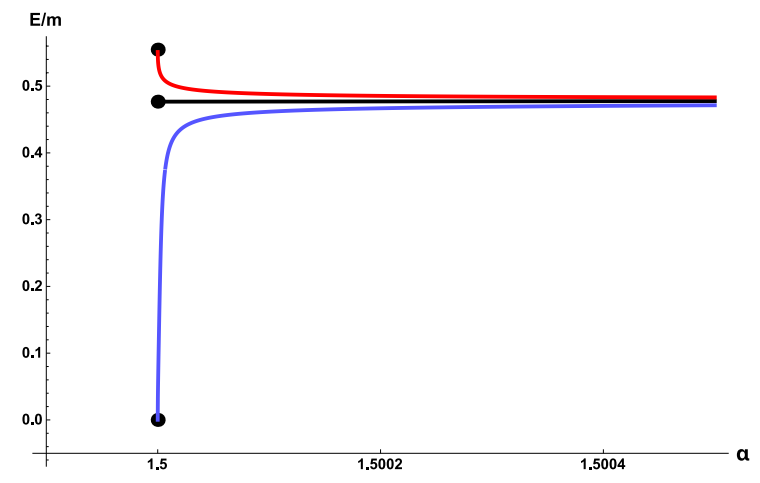

(b)

Figure 6. (a) Instability properties of the flow of $E_{n}^{I I}(\theta)$ for $\theta=\frac{\pi}{4}$ and $\theta_{ \pm}=\frac{\pi}{4} \pm 0.001$ (up/down) and $j=3 / 2(\Lambda=m / 10)$. (b) the same properties for the flow of $E_{n}^{I V}(\theta)$ for $\theta=\frac{\pi}{2}$ and $\theta_{ \pm}=$ $\frac{\pi}{2} \pm 0.005$ (up/down) and $j=3 / 2(\Lambda=m / 10)$.

whereas black points are the starting points of bound state energy levels for all other boundary conditions $\theta \neq \pi / 2$. The green levels again are isolated and create a barrier for all the others. Notice that for any $\theta \neq \pi / 2$ there are energy levels which emerge from the continuum for large enough values of the charge $\alpha$. In fact there is an infinity of them if we consider higher excited bound states. In figure 5 we just displayed one of those levels emerging from the continuum for each boundary condition).

The very special matching of subcritical and supercritical regimes at critical points is possible because the derivatives of the spectral curves at $\alpha_{c}^{2}=j^{2}$ are divergent $\left(\left.\partial_{\alpha} E_{n}(\theta)\right|_{\alpha_{c}}= \pm \infty\right)$, except for $\theta=\frac{\pi}{4}$ and $\theta=\frac{3 \pi}{4}$. For those special values of $\theta$ the $\alpha$ derivatives of the subcritical spectral curves are finite and do coincide with the $\alpha$-derivatives of the supercritical spectral curves reaching the same points for $\theta=\frac{\pi}{2}$ (green curves in figure 5) and $\theta=0$ (gray curves in figure 5), respectively. The specific value of the derivative is $\Lambda$ dependent. For all other values of $\theta$ those derivatives are $\pm \infty$.

In figures 6 we show the instability of the isolated flow curves. The central flux curves correspond, respectively to $\theta=\frac{\pi}{4}$ and $\theta=\frac{\pi}{2}$, while the others correspond to small perturbations of these curves, respectively $\theta=\frac{\pi}{4} \pm 0.001$ and $\theta=\frac{\pi}{2} \pm 0.005$. We can see how, when approaching to $\alpha^{2}=j^{2}$, the perturbed curves follow the isolated curves flow but eventually they are attracted by two different eigenvalues of $E_{n}^{I I I}(0)$.

For the lowest angular momentum $j=\frac{1}{2}$ the spectral flow is very similar (figure 7 ). The only remarkable difference is the absence of a regular sector, where the boundary condition is unique, for any value of the impurity charge $\alpha$. This implies that in graphene the choice of a boundary condition is always necessary, which requires the introduction of two extra parameters in the definition of the quantum system: a dimensionful scale $\Lambda$ and an angular variable $\theta$. This is in contrast with what happens in three dimensions where for low values of the charge $Z \alpha<\sqrt{3} / 2$ there is no need of fixing a boundary condition. The space of boundary conditions has the topology of an infinity cylinder $S^{1} \times \mathbb{R} \equiv \mathbb{R}^{2} \backslash\{0\}$ for any value of the impurity charge $\alpha$. 


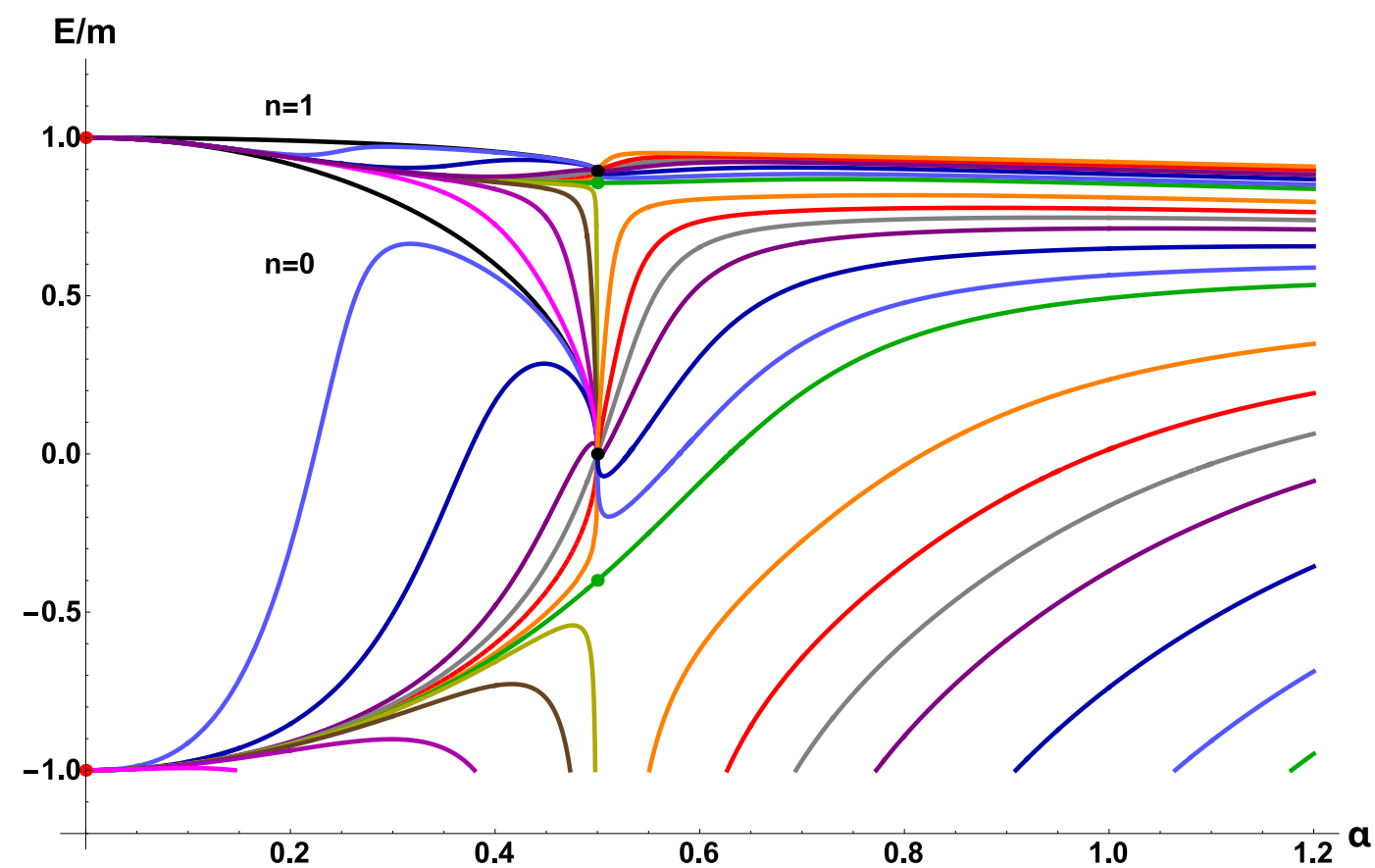

Figure 7. Flow of the lowest energy levels with angular momentum $j=1 / 2(\Lambda=m / 10)$ when the impurity charge crosses from subcritical regime to supercritical regime at $\alpha_{c}=1 / 2$. The colors correspond to different choices of boundary conditions. In the subcritical regime $\theta=0$ (hydrogen), $\theta=0.005 \pi, \theta=0.03 \pi, \theta=0.1 \pi, \theta=0.2 \pi, \theta=0.25 \pi$ (isolated), $\theta=0.3 \pi, \theta=0.5 \pi$ (meta-hydrogen), $\theta=0.75 \pi, \theta=0.9 \pi, \theta=0.99 \pi, \theta=0.999 \pi$; and in the supercritical regime $\theta=0, \theta=0.1 \pi, \theta=0.25 \pi, \theta=0.5 \pi$ (isolated), $\theta=0.6 \pi, \theta=0.75 \pi, \theta=0.9 \pi$. Notice that as in the case $j=\frac{3}{2}$ at the critical point $\alpha_{c}=\frac{1}{2}$ the tangent vectors to the spectral curves are vertical except for the green and gray curves which correspond to $\theta=\frac{\pi}{4}$ and $\theta=\frac{3 \pi}{4}$ in the subcritical regime and $\theta=\frac{\pi}{2}$ and $\theta=0$ supercritical regime, respectively.

\section{Conclusions}

In summary, the above analysis in terms of boundary conditions shows that in graphene we have infinite set of self adjoint Dirac operator for any $\alpha>0$ (for $j=\frac{1}{2}$ ) which are parameterized by an ultraviolet scale $\Lambda$ and an angle $\theta \in[0, \pi)$. In this sense the behaviour of impurities in graphene is different from that of hydrogenoid atoms in QED. The lowest angular momentum states of graphene are always in the subcritical regime unlike in the 3D hydrogen atom, which requires the introduction of appropriate boundary conditions that depend on a UV scale $\lambda$ and a dimensionless parameter $\theta$.

Unitarity is guaranteed for any value of the charge impurity $\alpha$. Even more, the parameters introduced by the boundary conditions $\Lambda, \theta$ that renormalize the singular UV of the impurity induce remarkable observable effects. The dependence on the choice of boundary conditions at the singularity defines a flow of energy levels. The analysis of the flow of boundary levels displays interesting physical properties. Changes of the $\theta$ parameter which characterizes the self adjoint extension of the Hamiltonian can pump each hydrogenoid level into the next one after a recursive loop in the parameter space recalling the pumping mechanism of topological insulators. A Berry phase can be also associated to this process. 
All energy levels in the hydrogenoid spectrum, except the fundamental one, are degenerate, but the introduction of the parameter $\theta$ breaks down this degeneracy. Moreover, it is possible to change, by adiabatic variations of $\alpha$, the energy levels from the subcritical to the supercritical regime in a continue way. Some bound states emerge (merge) from the continuum in this process. This is a consequence of the interesting properties of the RG flow for the subcritical and supercritical regime. Near the critical charge the energy levels are attracted by the points of the spectra of the Hamiltonian at the critical charge $\alpha_{c}^{2}=j^{2}$ and the particular value of the boundary conditions $\theta=0$. The attracting Hamiltonian corresponds to the hydrogenoid atom spectrum at the critical charge. Only few levels remain isolated in a unstable way. This points out that the critical charge $\alpha_{c}^{2}=j^{2}$ of the hydrogenoid case is not a singular case from the quantum physics viewpoint. The theory is well defined below and above this critical charge in the subcritical and supercritical regimes. The transition from the subcritical to the supercritical regime does not imply a critical change in the physical description of the system.

However the preservation of unitarity does not guarantees the stability of the theory in the supercritical regime because of the presence of a new infinite set of localized states with negative energies. Besides the standard stability analysis of the theory in absence of impurities, where all negative energy levels are filled by a Dirac sea of electrons, we have to add another sea of electrons to fill the infinite set of negative energy bound states. These infinities turn out to be finite when one recovers the discrete analysis on a graphene honeycomb lattice of finite size. However, the apparent stability of the theory pointed out by the careful analysis of the boundary conditions of the Hamiltonian can not hide that the physical behaviour of graphene is quite special in the supercritical phase. The fact that hydrogenoid energy levels become complex in the supercritical regime implies the presence of resonances in the spectral density of the scattering matrix in the positron (hole) channel. These resonances are also the root of bound states levels which emerge from the continuum negative spectrum $E<-m$ (see figure 4 ).

In the supercritical regime there is an infinite number of quasi-bound states embedded in the lower continuum $E<-m$ which are visible in the spectral density. If they are not filled when crossing the Fermi level $E=-m$, some normal electrons will jump into these empty levels generating particle/hole pairs. The positive charges will move to infinite and disappear whereas the negative charges remain localized near the impurity giving rise to a screening of the impurity charge. We have assumed a positively charged impurity but due to the $\mathrm{CP}$ invariance of the theory a similar phenomenon occurs for negative charged impurities.

The striking properties of the spectral flow discussed above can be experimentally tested by varying the impurity charge $\alpha$. This can be achieved by changing the dielectric properties of graphene environment [53]. The process will start with a supercritical impurity charge and then by changing the dielectric properties of the substrate it is possible to reduce the screening the effective coupling of the impurity to graphene electrons till reaching the critical charge and beyond that the subcritical regime. It will be very interesting see how the Coulomb supercritical extra screening disappears suddenly when crossing the critical charge barrier at $\alpha_{c}$. 
The phenomena described above are reminiscent of what happens in Quantum Eletrodynamics $[5,6]$. The main difference is that the value of the critical charge in graphene is $\alpha_{c}=j$ whereas in QED is $Z=137$, which is very hard to realize in Nature. The screening phenomenon due to supercritical pair creation has been recently observed in graphene [18] and in QED a similar phenomenon might be also observed in the heavy ions collisions (see [54] for an updated review). There is another remarkable difference between the two theories. In graphene for any value of $\alpha>0$ the system is in a subcritical regime at least in the lowest angular momentum sector $\left(j=\frac{1}{2}\right)$ which requires always the choice of an extra parameter to fix the boundary condition at the origin. However, in QED for $Z<137$, e.g. for the hydrogen atom the Hamiltonian is essentially selfadjoint in lowest angular momentum sector. Thus there is no need to fix the boundary condition at the origin. In particular a potential $\delta$ like perturbation has no effect in the spectrum. In particular this means that the relativistic interpretation of the Lamb effect cannot be understood in pure relativistic quantum mechanics and requires a full field theoretical analysis, unlike in the non-relativistic quantum mechanics approach.

The analysis of the energy spectrum in the gapless semi-metal regime of graphene can be carried out in a similar way. The boundary conditions are exactly the same as in the massive case and, thus, the different physical regimes are the same. However, there is a fundamental difference, there are no electronic bound states, because the exponential decay at infinity disappears when $m \rightarrow 0$, although there are some special points of the continuous spectrum that correspond to resonances which can be observed in scattering processes [18].

Open Access. This article is distributed under the terms of the Creative Commons Attribution License (CC-BY 4.0), which permits any use, distribution and reproduction in any medium, provided the original author(s) and source are credited.

\section{References}

[1] I. Pomeranchuk and Y. Smorodinsky, On energy level in systems with $Z>137$, J. Phys. (USSR) 9 (1945) 97.

[2] Y.B. Zeldovich and V.S. Popov, Electronic structure of superheavy atoms, Sov. Phys. Usp. 14 (1972) 673.

[3] V.S. Popov, On the properties of the discrete spectrum for Z close to 137, Sov. Phys. JETP 33 (1971) 665.

[4] V.S. Popov, Critical charge in quantum electrodynamics, Phys. Atom. Nucl. 64 (2001) 367.

[5] B. Müller, H. Peitz, J. Rafelski and W. Greiner, Solution of the Dirac equation for strong external fields, Phys. Rev. Lett. 28 (1972) 1235 [InSPIRE].

[6] V. Shabaev, A.I. Bondarev, D.A. Glazov, Y.S. Kozhedub, I.A. Maltsev, A.V. Malyshev et al., QED with heavy ions: on the way from strong to supercritical fields, PoS FFK2019 (2020) 052 [arXiv : 1910.01373] [INSPIRE].

[7] V.N. Gribov, ORSAY lectures on confinement. I, hep-ph/9403218 [INSPIRE].

[8] V.N. Gribov, Orsay lectures on confinement. II, hep-ph/9404332 [INSPIRE]. 
[9] V.N. Gribov, Orsay lectures on confinement. III, hep-ph/9905285 [INSPIRE].

[10] V. Gribov, QCD at large and short distances (annotated version), Eur. Phys. J. C 10 (1999) 71 [hep-ph/9807224] [INSPIRE].

[11] V.N. Gribov, Gauge Theories and Quark Confinement. Collection of works, Phasis Publishing House, Moscow (2002), 357.

[12] M. Asorey and A. Santagata, Instability of Coulomb phase in QCD, PoS ConfinementX (2012) 057 [INSPIRE].

[13] M. Asorey and A. Santagata, Coulomb phase stability and quark confinement, PoS QCD-TNT-III (2014) 004.

[14] M. Asorey and A. Santagata, Instabilities of Coulomb phases and quark confinement in QCD, AIP Conf. Proc. 1606 (2014) 407.

[15] M. Asorey and A. Santagata, Instabilities of Coulomb phases and the Gribov picture of confinement, Int. J. Mod. Phys. 31 (2016) 1645024.

[16] K.S. Novoselov, A.K. Geim, S.V. Morozov, D. Jiang, Y. Zhang, S.V. Dubonos, I.V. Gregorieva and A.A. Firsov, Electric Field Effect in Atomically Thin Carbon Films, Science 306 (2004) 666.

[17] A.H. Castro Neto, F. Guinea, N.M.R. Peres, K.S. Novoselov and A.K. Geim, The electronic properties of graphene, Rev. Mod. Phys. 81 (2009) 109 [arXiv:0709.1163] [InSPIRE].

[18] Y. Wang et al., Observing Atomic Collapse Resonances in Artificial Nuclei on Graphene, Science 340 (2013) 734.

[19] B.L. Voronov, D.M. Gitman and I.V. Tyutin, Dirac Hamiltonian with superstrong Coulomb field, Theor. Math. Phys. 150 (2007) 34 [quant-ph/0608221] [INSPIRE].

[20] T. Reichhardt,New form of hydrogen power provokes scepticism, Nature 404 (2000) 218.

[21] N. Dombey, The hydrino and other unlikely states, Phys. Lett. A 360 (2006) 62 [physics/0608095] [INSPIRE].

[22] G.W. Semenoff, Condensed Matter Simulation of a Three-dimensional Anomaly, Phys. Rev. Lett. 53 (1984) 2449 [INSPIRE].

[23] S. Zhou, G. Gweon and A. Fedorov, Substrate-induced bandgap opening in epitaxial graphene, Nature Mater. 6 (2007) 770.

[24] D.S. Novikov, Elastic scattering theory and transport in graphene, Phys. Rev. B 76 (2007) 245435 .

[25] A.V. Shytov, M.I. Katsnelson and L.S. Levitov, Vacuum Polarization and Screening of Supercritical Impurities in Graphene, Phys. Rev. Lett. 99 (2007) 236801.

[26] A.V. Shytov, M.I. Katsnelson and L.S. Levitov, Atomic Collapse and Quasi-Rydberg States in Graphene, Phys. Rev. Lett. 99 (2007) 246802.

[27] V.M. Pereira, J. Nilsson and A.H. Castro Neto, Coulomb Impurity Problem in Graphene, Phys. Rev. Lett. 99 (2007) 166802.

[28] R.R. Biswas, S. Sachdev and D.T. Son, Coulomb impurity in graphene, Phys. Rev. B 76 (2007) 205122 [arXiv:0706 . 3907] [INSPIRE].

[29] D.S. Novikov, Numbers of donors and acceptors from transport measurements in graphene, Appl. Phys. Lett. 91 (2007) 102102. 
[30] M.M. Fogler, D.S. Novikov and B.I. Shklovskii, Screening of a hypercritical charge in graphene, Phys. Rev. B 76 (2007) 233402.

[31] I.S. Terekhov, A.I. Milstein, V.N. Kotov and O.P. Sushkov, Screening of Coulomb impurities in graphene Phys. Rev. Lett. 100 (2008) 076803.

[32] V.M. Pereira, V.N. Kotov and A.H. Castro Neto, Supercritical Coulomb impurities in gapped graphene, Phys. Rev. B 78 (2008) 085101.

[33] K.S. Gupta and S. Sen, Bound states in gapped graphene with impurities: Effective low-energy description of short-range interactions, Phys. Rev. B 78 (2008) 205429 [arXiv:0808.2864] [INSPIRE].

[34] K.S. Gupta and S. Sen, Bound States in Graphene, Mod. Phys. Lett. A 24 (2009) 99 [arXiv:0805.3433] [INSPIRE].

[35] O.V. Gamayun, E.V. Gorbar and V.P. Gusynin, Supercritical Coulomb center and excitonic instability in graphene, Phys. Rev. B 80 (2009) 165429.

[36] O.V. Gamayun, E.V. Gorbar and V.P. Gusynin, Gap generation and semimetal-insulator phase transition in graphene, Phys. Rev. B 81 (2010) 075429 [arXiv:0911.4878] [InSPIRE].

[37] D. Kloepfer, A. De Martino and R. Egger, Bound States and Supercriticality in Graphene-Based Topological Insulators, Crystals 3 (2013) 14.

[38] V.N. Kotov, B. Uchoa, V.M. Pereira, A.H. Neto and F. Guinea, Electron-Electron Interactions in Graphene: Current Status and Perspectives, Rev. Mod. Phys. 84 (2012) 1067 [arXiv: 1012.3484] [INSPIRE].

[39] J. Wang, H.A. Fertig and G. Murthy, Critical behavior in graphene with Coulomb interactions, Phys. Rev. Lett. 104 (2010) 186401.

[40] K.M. Case, Singular potentials, Phys. Rev. 80 (1950) 797 [INSPIRE].

[41] V.R. Khalilov and C.-L. Ho, Dirac electron in a Coulomb field in (2+1)-dimensions, Mod. Phys. Lett. A 13 (1998) 615 [hep-th/9801012] [INSPIRE].

[42] M. Asorey, A.P. Balachandran and J.M. Pérez-Pardo, Edge States: Topological Insulators, Superconductors and QCD Chiral Bags, JHEP 12 (2013) 073 [arXiv:1308.5635] [INSPIRE].

[43] M. Asorey, A.P. Balachandran and J.M. Pérez-Pardo, Edge states at phase boundaries and their stability, Rev. Math. Phys. 28 (2016) 1650020.

[44] M. Asorey, A. Ibort and G. Marmo, The Topology and Geometry of self-adjoint and elliptic boundary conditions for Dirac and Laplace operators, Int. J. Geom. Meth. Mod. Phys. 12 (2015) 1561007 [arXiv:1510.08136] [INSPIRE].

[45] D.M. Gitman, A.D. Levin, I.V. Tyutin and B.L. Voronov, Electronic Structure of Superheavy Atoms. Revisited, Phys. Scripta 87 (2013) 038104.

[46] D.M. Gitman, I.V.Tyutin and B.L. Voronov, Self-adjoint Extensions in Quantum Mechanics. General theory and applications to Schrödinger and Dirac equations with singular potentials, Birkhäuser, Boston, U.S.A. (2012).

[47] M. Abramowitz and I.A. Stegun, Handbook of Mathematical Functions, Dover, New York, U.S.A. (1964).

[48] F.W.J. Olver et al., [DLMF] NIST Digital Library of Mathematical Functions, http://dlmf.nist.gov/, (2015). 
[49] R.B. Laughlin, Quantized Hall conductivity in two-dimensions, Phys. Rev. B 23 (1981) 5632 [INSPIRE].

[50] B.I. Halperin, Quantized Hall conductance, current carrying edge states, and the existence of extended states in a two-dimensional disordered potential, Phys. Rev. B 25 (1982) 2185 [INSPIRE].

[51] M. Asorey, A. Ibort and G. Marmo, Global theory of quantum boundary conditions and topology change, Int. J. Mod. Phys. A 20 (2005) 1001 [hep-th/0403048] [InSPIRE].

[52] C.L. Kane and E.J. Mele, Quantum Spin Hall Effect in Graphene, Phys. Rev. Lett. 95 (2005) 226801 [cond-mat/0411737] [INSPIRE].

[53] C. Jang, S. Adam, J.-H. Chen, E.D. Williams, S. Das Sarma and M.S. Fuhrer, Tuning the Effective Fine Structure Constant in Graphene: Opposing Effects of Dielectric Screening on Short- and Long-Range Potential Scattering, Phys. Rev. Lett. 101 (2008) 146805.

[54] J. Rafelski, J. Kirsch, B. Müller, J. Reinhardt and W. Greiner, Probing QED Vacuum with Heavy Ions, In New Horizons in Fundamental Physics, S. Schramm, M. Schäfer, eds., FIAS Interdisciplinary Science Series. Springer (2017). 